Bulletin de la Société Royale des Sciences de Liège, Vol. : 91, articles, 2021, p. 317 - 360

\title{
Contribution à l'étude de la végétation de certains massifs montagneux de la Kabylie des Babors (Nord-Est algérien)
}

\section{Contribution to the study of the vegetation of some mountains to the Kabylia of Babors (Northeastern Algeria).}

\author{
Manuscrit reçu le 21 octobre 2021 et accepté le 23 novembre 2021 \\ Mebarek BouchiBANE ${ }^{11}$, Mourad ZEMOURI ${ }^{1}$ \& Rachid TOUMI ${ }^{2}$
}

${ }^{1}$ Laboratoire d'Écologie et Environnement, Faculté des Sciences de la Nature et de la Vie. Université A. Mira de Bejaia, Targa Ouzemmour, 06000 Bejaia, Algérie.

${ }^{2}$ Laboratoire de gestion et valorisation des ressources naturelles et assurance qualité, Faculté des Sciences de la Nature et de la Vie et des sciences de la terre. Université AMO de Bouira, Algérie.

\section{Résumé}

Une étude floristique et phytogéographique a été éffectuée dans deux massifs montagneux de la Kabylie des Babors (Nord-Est algérien), dans l'objectif de conservation du patrimoine naturel. L'échantillonnage mené entre 2017 et 2020 au niveau des formations forestières et pré-forestières de Cèdre (Cedrus atlantica (Endl.) Carrière), de Chêne zéen (Quercus canariensis Willd.), de Chêne liège (Quercus suber L.) et des pelouses de montagne, s'est soldé par l'inventaire de 377 taxons appartenant à 246 genres et 65 familles de plantes vasculaires. L'analyse du spectre chorologique brut indique une dominance significative des espèces méditerranéennes (54,6\%). L'élément endémique ou sub-endémique est bien représenté $(14,6 \%)$, mais l'élément le plus remarquable est l'ensemble septentrional qui atteint $26,8 \%$ de la flore étudiée. L'analyse du spectre biologique global montre une dominance des hémicryptophytes $(43,8 \%)$ sur les autres formes de vie. Cette région constitue un refuge conservatoire pour une flore d'origine septentrionale et/ou endémique.

Mots-clés : flore, phytogéographie, endémisme, zone de refuge, conservation

\begin{abstract}
A floristic and phytogeographic study was carried out in two mountain ranges in the Kabylia of Babors (North-East of Algeria), with the objective of conservation of natural heritage. The sampling carried out at the level of forest and preforest formations of Cedar (Cedrus atlantica (Endl.) Quarry), Zeen oak (Quercus canariensis Willd.), Cork oak (Quercus suber L.) and mountain grasslands between 2017 and 2020 resulted in the inventory of 377 taxa belonging to 246 genera and 65 families of vascular plants. Analysis of the chorological spectrum
\end{abstract}

\footnotetext{
${ }^{1}$ Auteur pour correspondance : Téléphone : (213) 7975092 13, E-mail: mebarek_bouchibane@yahoo.fr
} 
indicates a significant dominance of Mediterranean species (54.6\%). The endemic or subendemic element is well represented (14.6\%), but the most remarkable element is the northern whole with $26.8 \%$ of the flora studied. Analysis of the global biological spectrum showed the dominance of hemicryptophytes $(43.8 \%)$ over the other life-forms. This area is an exceptional current refuge for northern and/or endemic flora.

Key words: flora, phytogeography, endemism, refuge area, conservation.

\section{Introduction}

Bien que mondialement reconnu comme l'un des principaux points chauds «hotspots» de biodiversité végétale, le bassin méditerranéen demeure encore méconnu en particulier sur ses rives sud et est (Médail \& Quézel, 1997 ; Véla, 2018). Cette région méditerranéenne abrite une diversité biologique de première importance (Amirouche \& Misset, 2009). Elle s'individualise par de nombreuses originalités environnementales et écologiques qui confèrent à sa biodiversité une valeur patrimoniale mondiale (Daki, 2004). La région méditerranéenne figure parmi les régions les plus diversifiées du monde sur le plan floristique, avec un peu plus de 25000 espèces (Quézel, 1985). Environ 50\% de ces taxons sont endémiques (Médail \& Quézel, 1997) et par contre le niveau de menaces d'origine anthropique est de plus en plus considérable (Benoit \& Comeau, 2005).

Certaines régions du bassin méditerranéen constituent des centres majeurs d'endémismes (Quézel \& Médail, 2003). C'est le cas de la région «Kabylies-Numidie-Kroumirie » (Véla \& Benhouhou, 2007) sur la rive sud de la méditerranée, qui habrite de nombreuses zones importantes pour les plantes, dont la Kabylie des Babors fait partie (Yahi et al., 2012; Benhouhou et al., 2018). Cette zone de la Kabylie des Babors (Nord-Est algérien), est l'une des régions les plus remarquables par sa richesse et l'originalité de sa flore (Quézel, 1956 ; Maire, 1926) de part des conditions climatiques exceptionnelles, associées à une topographie très accidentée.

Parmi les travaux consacrés à la flore de la région de la Kabylie des Babors, on note ceux de : Battandier (1888-1890), Battandier \& Trabut (1895), Lapie (1914), Quézel (1956) et Gharzouli (2007). Malgré la grande richesse florisitique de ce secteur biogéographique, la biodiversité végétale de certaines localités reste encore moins connue (Chelli-Tabti et al., 2020). Les explorations récentes menées dans la région de la Kabylie des Babors, notamment dans diverses formations forestières en hautes montagnes, ont délivré d'importantes découvertes floristiques. C'est le cas de la récente découverte d'une d'orchidée nouvelle pour la flore d'Algérie et d'Afrique du Nord, Epipactis microphylla (Ehrh.) Sw. Au mont Babor (Bougaham et al., 2020) et d'une fougère Christella dentata Forssk. (Rebbas et al., 2019).

Si certains djebels (montagnes) de la Kabylie des Babors, ont fait l'objet d'études récentes telles que les monts de Tababort, Babor (Gharzouli, 2007), Sidi-Djaber et Adrar N'fad (Bouchibane et al., 2017), d'autres massifs comme celui d'Agouf (1120 m alt.) et le massif Adrar Ou-Mellal (1773 m alt.) n'ont pas été récemment explorés. Les études floristiques 
réalisées dans ces massifs, remontent à très longtemps (Quézel, 1956 ; Quézel \& Santa, 19621963).

Cette étude s'inscrit dans la continuité des études réalisées récemment dans cette région pour actualiser et affiner les connaissances floristiques et phytogéographiques de ce vaste territoire (Kabylie des Babors), en particulier dans les massifs montagneux d'Agouf et d'Adrar OuMellal, et pour évaluer les menaces qui pèsent sur elles ; autant de paramètres importants à la réflexion et à la mise en place de mesures visant leur conservation, protection et valorisation. L'une des finalités de cette étude est également d'évoquer l'intérêt patrimonial de quelques taxons endémiques et /ou rares de cette région des Babors.

\section{Matériel et méthodes}

\subsection{Site d'étude}

La chaine des Babors se situe entre le littoral de Bejaia au nord, et les plaines Sétifiennes au sud. À l'ouest elle est limitée par la vallée de la Soummam, à l'est par une ligne qui va d'El Aouana à Oued Djendjen, et au sud par Texenna (Figure 1).

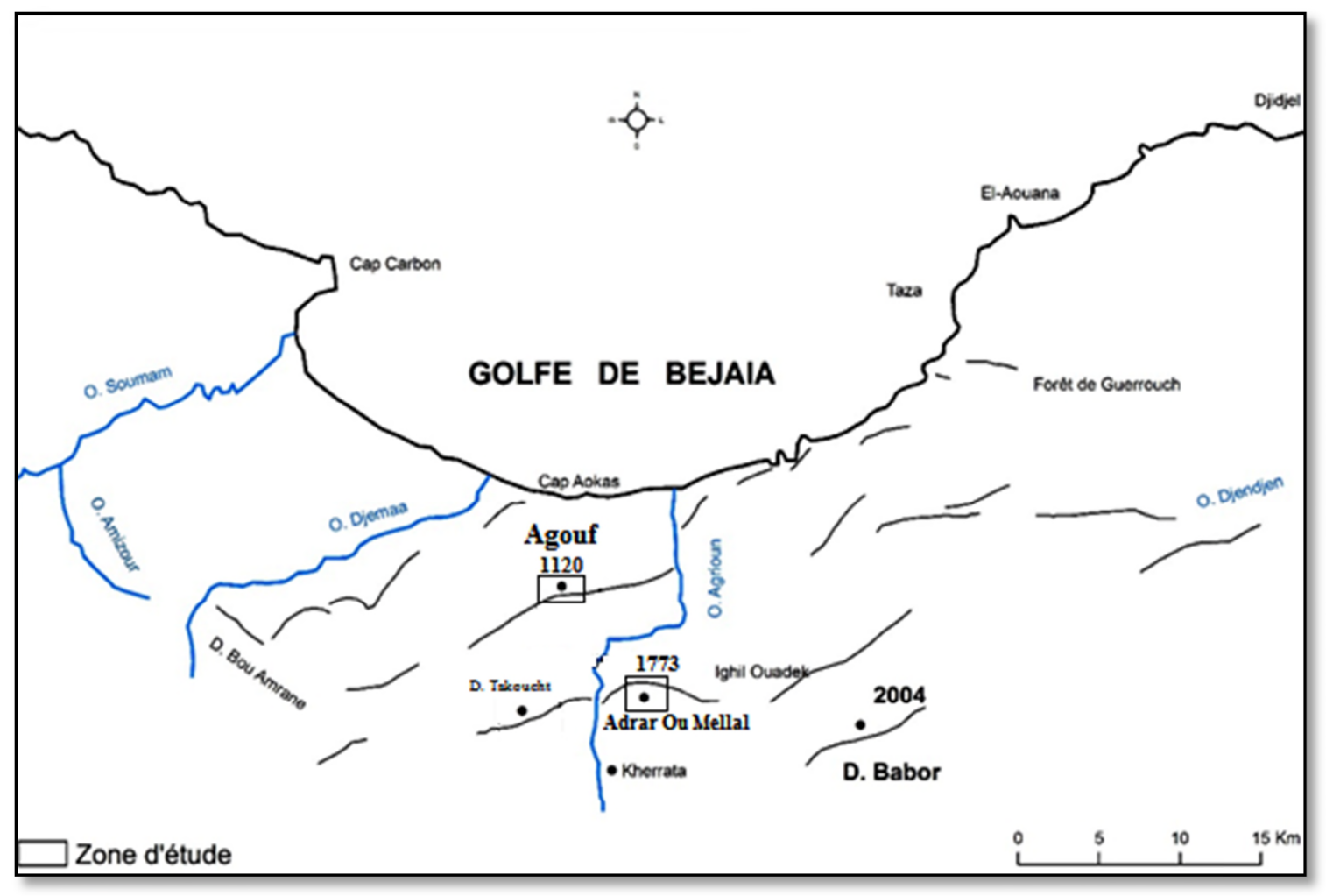

Figure 1 : Carte schématique de la localisation géographique de la zone d'étude.

(Coordonnée du point central de la carte : $36^{\circ} 38^{\prime} \mathrm{N} / 5^{\circ} 17^{\prime} \mathrm{E}$ )

Cette région est constituée de djebels organisés en chaînons sensiblement parallèles (Duplan, 1952). Cette étude concerne les massifs de moyennes altitudes, formés par le djebel Agouf 
$(1120 \mathrm{~m})$ au nord et le djebel Adrar Ou-Mellal $(1773 \mathrm{~m})$ plus au sud. Le djebel Agouf est constitué de calcaires liasiques, alors que le massif Adrar Ou-Mellal est formé par les calcaires dolomités et minéralisés du Lias inférieur surmontés par les calcaires du Lias moyen qui forment les crêtes (Duplan, 1952).

Les précipitations et les températures des massifs étudiés sont obtenues par extrapolation à partir des données relevées de la station météorologique de Kherrata (470 m d'altitude) sur une période de 15 ans (1996 - 2010). Cette extrapolation a été faite grâce à une méthode de correction proposée par Seltzer (1946) qui a déterminé pour les précipitations un gradient de $40 \mathrm{~mm}$ pour $100 \mathrm{~m}$ de dénivelé. L'abaissement des températures pour une élévation de $100 \mathrm{~m}$ d'altitude est de $0,7{ }^{\circ} \mathrm{C}$ pour les maximales et de $0,4{ }^{\circ} \mathrm{C}$ pour les minimales (Seltzer, 1946). De ce fait, les précipitations moyennes annuelles sont estimées à 1415,8 $\mathrm{mm}$ au sommet du massif d'Adrar Ou-Mellal et à 1154,6 mm au sommet du djebel Agouf (Tableau 1). Les températures maximales du mois le plus chaud sont estimées à $25,1^{\circ} \mathrm{C}$ au sommet du massif d'Adrar Ou-Mellal $(1773 \mathrm{~m})$ et à 29,6 ${ }^{\circ} \mathrm{C}$ au sommet du djebel Agouf $(1120 \mathrm{~m})$, alors que celles des minima du mois le plus froid sont estimées à $1,4{ }^{\circ} \mathrm{C}$ et à $-1,2{ }^{\circ} \mathrm{C}$ pour le djebel Agouf et le djebel Adrar Ou-Mellal respectivement (Tableau 1). Le Djebel Adrar Ou-Mellal $(1773 \mathrm{~m})$ appartient ainsi à l'étage bioclimatique per-humide à hiver froid (sensu Emberger, 1955), par contre, le djebel Agouf (1120 m) appartient à l'étage bioclimatique humide à hiver frais (sensu Emberger, 1955). La durée de la période sèche (période de l'année ou la somme des précipitations est inférieure à deux fois la moyenne des températures : $\mathrm{P}<2 \mathrm{~T}$ ) estimée inférieure à 3 mois et la fréquence du brouillard au niveau des versants de ces massifs, surtout durant la période estivale, atténuent l'intensité de la sécheresse dans ces massifs.

Tableau 1 : Données climatiques dans la région d'étude (station météorologique de Kherrata et formules d'extrapolation selon Seltzer 1946). Alt. (altitude); P (cumul des précipitations annuelles); m (moyenne des minima du mois le plus froid) ; M (moyenne des maxima du mois le plus chaud) ; Q (indice climatique d'Emberger); EB (étages bioclimatiques sensu Emberger, 1955).

\begin{tabular}{|l|c|c|c|c|c|l|}
\hline \multicolumn{1}{|c|}{ Localité } & Alt. $(\mathbf{m})$ & $\mathbf{P}(\mathbf{m m})$ & $\mathbf{M}\left({ }^{\circ} \mathbf{C}\right)$ & $\mathbf{m}\left({ }^{\circ} \mathbf{C}\right)$ & $\mathbf{Q}$ & \multicolumn{1}{|c|}{$\mathbf{E B}$} \\
\hline Kherrata & 470 & 894,6 & 34,2 & 4,0 & 101,6 & Sub-humide \\
\hline Agouf & 1120 & 1154,6 & 29,6 & 1,4 & 140,4 & Humide \\
\hline Adrar Ou-Mellal & 1773 & 1415,8 & 25,1 & $-1,2$ & 184,6 & Per-humide \\
\hline
\end{tabular}

La Kabylie des Babors, appartient au domaine méditerranéen Nord-africain (Quézel, 1978). Ce domaine méditerranéen Nord-Africain comprend plusieurs secteurs, eux-mêmes subdivisés en districts. Toute la partie nord se situe dans le secteur numidien, au niveau du district de petite Kabylie (Maire, 1926 ; Quézel \& Santa, 1962-1963).

\subsection{Méthodologie}

Des relevés phytoécologiques ont été éffectués au niveau des formations forestières et préforestières de Cèdre (Cedrus atlantica (Endl.) Carrière), de Chêne zéen (Quercus 
canariensis Willd.), de Chêne liège (Quercus suber L.) et des pelouses de montagne, selon un échantillonnage systèmatique afin d'établir une liste globale des espèces. L'échantillonnage s'étale du mois de mars 2017 au mois de juin 2020. 90 relevés ont été réalisés sur une surface variable dite « aire minimale » (Gounot, 1969), allant de $40 \mathrm{~m}^{2}$ pour les pelouses à $80 \mathrm{~m}^{2}$ pour les matorrals et jusqu'à $280 \mathrm{~m}^{2}$ en contexte forestier. Des spécimens d'herbier ont été récoltés pour validation ainsi que des prises de photos. Ces spécimens d'herbier sont déposés au laboratoire de Recherche en Ecologie et Environnement de l'Université de Bejaia. L'identification des taxons a été faite à l'aide des flores de Battandier (1888-1890), Battandier \& Trabut (1895), Maire (1952-1987) et Quézel \& Santa (1962-1963). La nomenclature retenue a été actualisée selon Dobignard \& Chatelain (2010-2013) et l'African Plant Database (APD, 2021). Sur le plan phytogéographique, notre travail fait référence aux indications de Pignatti (1982), Jeanmonod \& Gamisans (2007), Fennane et al. (2014) and the Euro+Med Plant Base (2006-2021). L'information sur les espèces endémiques est issue de Dobignard \& Chatelain (2010-2013). Les types biologiques (sensu Raunkiaer, 1934) des différents taxons ont été décrits à partir d'observations de terrain et des ouvrages de Pignatti (1982), Gharzouli (2007), Jeanmonod \& Gamisans (2007) et de Fennane et al. (2014). La liste des espèces menacées présentes sur le site a été réalisée sur la base de critères de rareté établis par Quézel \& Santa (1962-1963) et de vulnérabilité à l'échelle globale établis par l'Union Internationale de la Conservation de la Nature en 1997 (Walter \& Gillett, 1998). La liste rouge produite permet de mettre en évidence les taxons à plus haut risque d'extinction et de définir les priorités dans les politiques de sauvegarde et de conservation de la biodiversité végétale. Nous avons aussi considéré comme espèces d'intérêt patrimonial les espèces protégées par le Décret $\mathrm{n}^{\circ}$ 03-12/12-28 complétant la liste des espèces végétales non cultivées protégées en Algérie (J.O.R.A. 2012).

\section{Résultats}

\subsection{Diversité floristique}

La liste floristique obtenue sur les 90 placettes, comprend 65 familles de plantes vasculaires (phanérogames et cryptogames vasculaires), 246 genres et 377 taxons. Chaque relevé compte en moyenne 25 taxons. Sur les 90 relevés effectués, 55 ont été réalisés au massif Adrar OuMellal et 35 au djebel Agouf. Les familles les mieux représentées sont les Asteraceae avec 44 taxons et 36 genres, suivies par les Fabaceae (35 taxons et 20 genres), les Poaceae (33 taxons et 25 genres), les Orchidaceae (21 taxons et 9 genres), les Lamiaceae (20 taxons et 15 genres), les Apiaceae (18 taxons et 16 genres) et les Caryophyllaceae (16 taxons et 10 genres). Ces sept familles représentent à elles seules 49,6\% de la flore analysée. 16 familles sont représentées par un seul taxon. L'ensemble des espèces inventoriées est repris en annexe.

\subsection{Spectre chorologique}

L'analyse du spectre chorologique global (Tableau 2), indique que la flore analysée appartient à plusieurs ensembles phytogéographiques. Le mieux représenté est l'ensemble méditerranéen 
avec 206 taxons, soit 54,6\% de la flore répertoriée, ce qui correspond à 52 (80,0\%) familles. Les mieux représentées sont les Asteraceae (30 taxons), suivies par les Fabaceae (28 taxons), les Poaceae (15 taxons), les Lamiaceae (12 taxons), les Rosaceae et les Apiaceae avec 8 taxons chacune.

L'ensemble septentrional (nordique) arrive en seconde position avec ses 101 taxons (26,8\%). 42 familles $(64,6 \%)$ présentent des espèces nordiques. Les plus riches sont les Poaceae avec 12 taxons, suivies par les Rosaceae et les Brassicaceae avec 7 taxons chacune, suivies par les Orchidaceae avec 6 taxons, suivies par les Fabaceae et les Asteraceae (5 taxons).

L'ensemble endémique occupe la troisième place avec 55 taxons endémiques régionaux (Tableau 3) : 14 taxons endémiques algériens stricts (3,7\%), 13 endémiques algéro-tunisiens (3,5\%), 10 endémiques algéro-marocains $(2,7 \%)$ et 18 endémiques nord-africaines $(4,8 \%)$, soit $14,6 \%$ de la flore inventoriée. 24 familles $(36,9 \%)$ présentent des taxons endémiques et les Asteraceae sont les mieux représentées avec 7 endémiques, les Apiaceae (6 endémiques), les Lamiaceae, les Orchidaceae et les Caryophyllaceae avec 5 endémiques chacune, suivie par les Primulaceae avec 3 endémiques. Enfin, l'ensemble à large répartition est représenté seulement par 15 taxons, soit 4,0\% de la flore recensée, appartenant à douze familles. Les cosmopolites et les subcosmopolites comptent 10 taxons (2,7\%). Par contre, le sous-élément tropical est représenté par 5 espèces $(1,3 \%)$.

Tableau 2 : Spectre chorologique global.

\begin{tabular}{|l|c|c|}
\hline Ensemble chorologique & Nombre d'espèces & Proportion (\%) \\
\hline \hline Méditerranéen & $\mathbf{2 0 6}$ & $\mathbf{5 4 , 6}$ \\
Stenoméditerranéen & 76 & 20,2 \\
Euryméditerranéen & 55 & 14,6 \\
Méditerranéen-montagnard & 09 & 2,4 \\
Atlantique-Méditérranéen & 13 & 3,4 \\
Méditerranéen-Touranien & 04 & 1,1 \\
Méditerranéenne S.S & 39 & 10,3 \\
Oro- Méditerranéenne & 09 & 2,4 \\
Méditerranéen-asiatique & 01 & 0,3 \\
\hline Septentrional & $\mathbf{1 0 1}$ & $\mathbf{2 6 , 8}$ \\
Eurasiatique & 40 & 10,9 \\
Paléotempéré & 23 & 6,1 \\
Européen & 20 & 5,3 \\
Europe-Caucase & 03 & 0,8 \\
Boréal & 07 & 1,9 \\
Circumboréal & 05 & 1,3 \\
Europe-Sibérie & 02 & 0,5 \\
Atlantique & 01 & 0,3 \\
\hline Endémique & $\mathbf{5 5}$ & $\mathbf{1 4 , 6}$ \\
Endémiques algériennes strictes & 14 & 3,7 \\
Endémiques algéro-tunisiennes & 13 & 3,5 \\
\hline
\end{tabular}


Bulletin de la Société Royale des Sciences de Liège, Vol. : 91, articles, 2021, p. 317 - 360

\begin{tabular}{|l|c|c|}
\hline Endémiques algéro-marocaines & 10 & 2,7 \\
Endémiques Nord-Africaines & 18 & 4,7 \\
& & \\
\hline Large répartition & $\mathbf{1 5}$ & $\mathbf{4 , 0}$ \\
Cosmopolite et subcosmopolite & 10 & 2,7 \\
Tropical et subtropical & 5 & 1,3 \\
\hline
\end{tabular}

Tableau 3 : Espèces endémiques observées au niveau des massifs étudiés.

\begin{tabular}{|c|c|c|c|}
\hline Taxons & Endémisme & Taxons & Endémisme \\
\hline $\begin{array}{l}\text { Ammoides atlantica (Coss. } \\
\text { \& Durieu) H. Wolff. }\end{array}$ & End Alg & $\begin{array}{l}\text { Epimedium perralderianum } \\
\text { Coss. }\end{array}$ & End Alg \\
\hline $\begin{array}{l}\text { Androrchis patens (Desf.) } \\
\text { D. Tyteca \& E. Klein. }\end{array}$ & $\begin{array}{l}\text { End Alg- } \\
\text { Tun }\end{array}$ & Erodium battandieranum Rouy & End Alg \\
\hline $\begin{array}{l}\text { Androrchis pauciflora } \\
\text { subsp. laeta (Steinh.) Véla, } \\
\text { Rebbas \& R. Martin }\end{array}$ & $\begin{array}{l}\text { End Alg- } \\
\text { Tun }\end{array}$ & Fritillaria oranensis Pomel & End N.Afr. \\
\hline $\begin{array}{l}\text { Arabis pubescens (Desf.) } \\
\text { Poir. }\end{array}$ & End N.Afr. & $\begin{array}{l}\text { Geranium atlanticum Boiss. \& } \\
\text { Reut. }\end{array}$ & End N.Afr. \\
\hline $\begin{array}{l}\text { Bromopsis erecta subsp. } \\
\text { microchaeta (Font Quer) H. } \\
\text { Scholz \& Valdés }\end{array}$ & $\begin{array}{l}\text { End Alg- } \\
\text { Mar }\end{array}$ & Hedera algeriensis Hibberd. & $\begin{array}{l}\text { End Alg- } \\
\text { Tun }\end{array}$ \\
\hline Bupleurum montanum Coss. & $\begin{array}{l}\text { End Alg- } \\
\text { Mar }\end{array}$ & $\begin{array}{l}\text { Helianthemum } \\
\text { helianthemoides } \\
\text { Grosser }\end{array}$ & End N.Afr. \\
\hline Carthamus multifidus Desf & End N.Afr. & $\begin{array}{l}\text { Heracleum sphondylium } \\
\text { subsp. algeriense (Batt. \& } \\
\text { Trab.) Dobignard }\end{array}$ & End Alg \\
\hline $\begin{array}{l}\text { Campanula trachelium L. } \\
\text { subsp. mauritanica (Pomel) } \\
\text { Quézel }\end{array}$ & $\begin{array}{l}\text { End Alg- } \\
\text { Mar }\end{array}$ & $\begin{array}{l}\text { Hieracium pseudopilosella } \\
\text { (Ten.) Sojak }\end{array}$ & End N.Afr. \\
\hline Catananche montana Coss. & $\begin{array}{l}\text { End Alg- } \\
\text { Mar }\end{array}$ & Hippocrepis atlantica Ball & $\begin{array}{l}\text { End Alg- } \\
\text { Mar }\end{array}$ \\
\hline $\begin{array}{l}\text { Cedrus atlantica (Endl.) } \\
\text { Carriere }\end{array}$ & $\begin{array}{l}\text { End Alg- } \\
\text { Mar }\end{array}$ & $\begin{array}{l}\text { Iberis atlantica (Litard.\& } \\
\text { Maire) Greuter \& Burdet }\end{array}$ & $\begin{array}{l}\text { End Alg- } \\
\text { Mar }\end{array}$ \\
\hline $\begin{array}{l}\text { Cerastium atlanticum } \\
\text { Durieu }\end{array}$ & End N.Afr. & Iris inguicularis Poir. & $\begin{array}{l}\text { End Alg- } \\
\text { Tun }\end{array}$ \\
\hline $\begin{array}{l}\text { Conopodium glaberimum } \\
\text { (Desf.) Engstrand. }\end{array}$ & End N.Afr. & Knautia mauritanica Pomel & End N.Afr. \\
\hline $\begin{array}{l}\text { Coronilla valentina L. } \\
\text { subsp. speciosa (Uhrova) } \\
\text { Greuter \& Burdet }\end{array}$ & End Alg & $\begin{array}{l}\text { Linaria virgata Desf. subsp. } \\
\text { algeriensis Murb. }\end{array}$ & $\begin{array}{l}\text { End Alg- } \\
\text { Tun }\end{array}$ \\
\hline Cyclamen africanum Boiss. & End & Plagius maghrebinus Vogt \& & End N.Afr. \\
\hline
\end{tabular}


Bulletin de la Société Royale des Sciences de Liège, Vol. : 91, articles, 2021, p. 317 - 360

\begin{tabular}{|c|c|c|c|}
\hline \& Reut. & Tun & Greuter & \\
\hline $\begin{array}{l}\text { Cynosurus balansae Coss. } \\
\text { \& Durieu }\end{array}$ & $\begin{array}{l}\text { End Alg- } \\
\text { Mar }\end{array}$ & Lonicera kabylica Rehder & End Alg \\
\hline $\begin{array}{l}\text { Doronicum plantaginum } \\
\text { subsp. atlanticum (Chabert) } \\
\text { Rouy. }\end{array}$ & End N.Afr. & Lysimachia cousiniana Coss. & $\begin{array}{l}\text { End Alg- } \\
\text { Tun }\end{array}$ \\
\hline $\begin{array}{l}\text { Moehringia stellarioides } \\
\text { Coss. }\end{array}$ & End Alg & $\begin{array}{llll}\text { Sedum } & \text { multiceps } & \text { Coss. } & \& \\
\text { Durieu } & & & \end{array}$ & End Alg \\
\hline $\begin{array}{l}\text { Ophrys battandieri } \mathrm{G} . \\
\text { Camus }\end{array}$ & End N.Afr. & Sedum pubescens Vahl. & $\begin{array}{l}\text { End Alg- } \\
\text { Tun }\end{array}$ \\
\hline $\begin{array}{l}\text { Ophrys numida Devillers- } \\
\text { Tersch. \& Devillers }\end{array}$ & $\begin{array}{l}\text { End Alg- } \\
\text { Tun }\end{array}$ & $\begin{array}{l}\text { Senecio perralderianus Coss. } \\
\text { \& Durieu }\end{array}$ & End Alg \\
\hline $\begin{array}{l}\text { Origanum vulgare L. subsp. } \\
\text { glandulosum (Desf.) } \\
\text { Letswaart }\end{array}$ & $\begin{array}{l}\text { End Alg- } \\
\text { Tun }\end{array}$ & $\begin{array}{l}\text { Selinopsis montana Coss. \& } \\
\text { Durieu ex Batt. }\end{array}$ & $\begin{array}{l}\text { End } \\
\text { Tun }\end{array}$ \\
\hline $\begin{array}{l}\text { Paeonia mascula (L.) Mill. } \\
\text { subsp. atlantica (Cosson.) } \\
\text { Greuter \& Burdet }\end{array}$ & End Alg & $\begin{array}{l}\text { Silene atlantica Coss. \& } \\
\text { Durieu }\end{array}$ & $\begin{array}{l}\text { End Alg- } \\
\text { Tun }\end{array}$ \\
\hline $\begin{array}{l}\text { Paronychia arabica subsp. } \\
\text { aurasiaca (Coss.) Batt. }\end{array}$ & End N.Afr. & Silene choulettii Coss. & End Alg \\
\hline $\begin{array}{l}\text { Patzkea durandoi } \\
\text { (Clausson) G. H. Loos. }\end{array}$ & End N.Afr. & $\begin{array}{l}\text { Taraxacum inaequilobum } \\
\text { Pomel }\end{array}$ & End N.Afr. \\
\hline Phlomis bovei de Noé & $\begin{array}{l}\text { End Alg- } \\
\text { Tun }\end{array}$ & Teucrium atratum Pomel & End Alg \\
\hline $\begin{array}{l}\text { Pimpinella battandieri } \\
\text { Chabert }\end{array}$ & End Alg & $\begin{array}{l}\text { Thymus algériensis Boiss. \& } \\
\text { Reut. }\end{array}$ & End N.Afr. \\
\hline $\begin{array}{l}\text { Primula acaulis L. subsp. } \\
\text { atlantica (Maire \& } \\
\text { Wilczek) Greuter \& Burdet }\end{array}$ & $\begin{array}{l}\text { End Alg- } \\
\text { Mar }\end{array}$ & Thymus dreatensis Batt. & End Alg \\
\hline $\begin{array}{l}\text { Ranunculus spicatus } \\
\text { subsp. maroccanus (Coss.) } \\
\text { Greuter \& Burdet }\end{array}$ & $\begin{array}{l}\text { End Alg- } \\
\text { Mar }\end{array}$ & $\begin{array}{l}\text { Dactylorhiza munbyana } \\
\text { (Boiss. \& Reut.) Aver. }\end{array}$ & End N.Afr. \\
\hline $\begin{array}{l}\text { Viola mumbyana Boiss. \& } \\
\text { Reut. }\end{array}$ & End N.Afr. & & \\
\hline
\end{tabular}

\subsection{Spectre biologique global}

L'analyse du spectre biologique brut (Tableau 4) révèle que les hémicryptophytes sont prédominantes sur les autres formes de vie avec 165 taxons $(43,8 \%)$, suivies par les thérophytes avec 86 espèces $(22,8 \%)$. Les géophytes se positionnent à la troisième place avec 51 espèces $(13,5 \%)$. Les phanérophytes et les chaméphytes sont représentés respectivement par 48 taxons $(12,7 \%)$ et 27 taxons $(7,2 \%)$. 
Tableau 4 : Spectre biologique global.

\begin{tabular}{|l|c|c|}
\hline \multicolumn{1}{|c|}{ Types biologiques } & Nombre d'espèces & Proportion (\%) \\
\hline \hline Hémicryptophytes & 165 & 43,8 \\
Thérophytes & 86 & 22,8 \\
Géophytes & 51 & 13,5 \\
Phanérophytes & 48 & 12,7 \\
Chaméphytes & 27 & 07,2 \\
\hline
\end{tabular}

\subsection{Les espèces rares et menacées}

La flore analysée est composée de 114 (30,2\%) espèces rares sensu Quézel \& Santa (19621963), dont 10 très rares, 64 rares et 40 assez rares. Parmi ces taxons rares, 24 sont endémiques, 45 sont des espèces septentrionales, 44 sont des taxons méditerranéens et une espèce de l'élément large répartition. La liste rouge 1997 de l'UICN (Walter \& Gillett, 1998), comporte 64 espèces algériennes rares et menacées, parmi elles, 10 sont présentes dans nos relevés (Tableau 5), soit 15,6\%. En outre, 29 taxons inventoriés se retrouvent sur la liste des espèces végétales non cultivées et protégées (D.E. 2012).

Tableau 5 : Liste des espèces rares, menacées et protégées de la forêt des massifs étudiés d'après les données bibliographiques (Quézel \& Santa, 1962-63 ; Décret exécutif (D.E. 2012). U.I.C.N., 1997 (Walter \& Gillett, 1998). E : en danger, V : vulnérable. AR : Assez rare, R : Rare, RR : Très rare. AC : Assez commun.

\begin{tabular}{|c|c|c|c|}
\hline Taxons & $\begin{array}{c}\text { Quézel \& Santa } \\
(1962-1963) \\
\end{array}$ & D.E. (2012) & $\begin{array}{l}\text { UICN (Walter } \\
\text { \& Gillet, 1998) } \\
\end{array}$ \\
\hline Acer obtusatum Waldst. \& Kit. & $\mathrm{R}$ & Protégé & \\
\hline $\begin{array}{l}\text { Anacamptis coriophora subsp. } \\
\text { fragrans (Pollini). R.M. Bateman, } \\
\text { Pridgeon \& M.W. Chase }\end{array}$ & $\mathrm{AC}$ & Protégé & \\
\hline $\begin{array}{l}\text { Anacamptis morio subsp. longicornu } \\
\text { (Poir.) H. Kretzschmar, Eccarius \& } \\
\text { H.Dietr. }\end{array}$ & $\mathrm{R}$ & Protégé & \\
\hline $\begin{array}{l}\text { Anacamptis papilionacea (L.) R.M. } \\
\text { Bateman, Pridgeon \& M.W. Chase }\end{array}$ & $\mathrm{AR}$ & Protégé & \\
\hline $\begin{array}{l}\text { Androrchis olbiensis (Reut. ex Gren.) } \\
\text { D. Tyteca \& E. Klein. }\end{array}$ & $\mathrm{AR}$ & Protégé & \\
\hline $\begin{array}{l}\text { Androrchis patens (Desf.) D. Tyteca \& } \\
\text { E. Klein. }\end{array}$ & AR & Protégé & \\
\hline Campanula trichocalycina Ten. & $\mathrm{R}$ & Protégé & \\
\hline Cedrus atlantica (Endl.) Carrière & $\mathrm{AC}$ & Protégé & \\
\hline Cyclamen africanum Boiss. \& Reut. & $\mathrm{AC}$ & Protégé & \\
\hline Epimedium perralderianum Coss. & $\mathrm{RR}$ & Protégé & E \\
\hline Erodium battandieranum Rouy & $\mathrm{R}$ & Protégé & $\mathrm{R}$ \\
\hline Helianthemum helianthemoides Desf. & RR & Protégé & \\
\hline
\end{tabular}




\begin{tabular}{|l|c|c|c|}
\hline $\begin{array}{l}\text { Juniperus phoenicea L. subsp. } \\
\text { turbinata (Guss.) Arcang. }\end{array}$ & $\mathrm{AC}$ & Protégé & \\
\hline Lonicera kabylica (Batt.) Rehder & $\mathrm{R}$ & Protégé & $\mathrm{R}$ \\
\hline $\begin{array}{l}\text { Lysimachia cousiniana Coss \& Durieu } \\
\text { Moehringia stellarioides Coss. }\end{array}$ & $\mathrm{AC}$ & Protégé & \\
\hline $\begin{array}{l}\text { Orchis purpurea Huds. subsp. } \\
\text { purpurea }\end{array}$ & $\mathrm{R}$ & Protégé & $\mathrm{R}$ \\
\hline Phlomis bovei De Noé & $\mathrm{R}$ & Protégé & \\
\hline Pimpinella battandieri Chabert & $\mathrm{R}$ & Protégégé & $\mathrm{R}$ \\
\hline $\begin{array}{l}\text { Primula acaulis subsp. atlantica } \\
\text { (Maire \& Wilczek) Greuter \& Burdet }\end{array}$ & $\mathrm{R}$ & Protégé & \\
\hline Sedum multiceps Coss. \& Durieu & $\mathrm{R}$ & Protégé & $\mathrm{R}$ \\
\hline $\begin{array}{l}\text { Selinopsis } \text { montana } \text { Coss. \& Durieu ex } \\
\text { Batt. }\end{array}$ & $\mathrm{AR}$ & Protégé & $\mathrm{R}$ \\
\hline Sorbus aria Crantz & $\mathrm{R}$ & Protégé & \\
\hline Sorbus torminalis (L.) Crantz & $\mathrm{R}$ & Protégé & \\
\hline Taxus baccata L. & $\mathrm{AR}$ & Protégé & \\
\hline Teucrium atratum Pomel & $\mathrm{R}$ & Protégé & $\mathrm{R}$ \\
\hline Thymus dreatensis Batt. & $\mathrm{R}$ & Protégé & $\mathrm{V}$ \\
\hline Viburnum lantana L. & $\mathbf{R R}$ & Protégé & \\
\hline \multicolumn{1}{|c|}{ Total des taxons } & $\mathbf{2 9}$ & $\mathbf{1 0}$ \\
\hline
\end{tabular}

\section{Discussion}

\subsection{Diversité floristique}

Le nombre de taxons recensé (377 espèces et sous espèces) est important, mais n'est pas exhaustif des massifs étudiés, car certains endroits d'accès très difficile n'ont pas été explorés. Ce nombre de taxons (377) représente 12,0\% de la flore d'Algérie du nord estimée à 3150 taxons par Médail \& Quézel (1997). Il est inférieur à ceux donné par Meddour (2010) pour les groupements forestiers et préforestiers de la Kabylie Djurdjureenne (500 taxons) et par Messaoudene et al. (2007) pour la forêt d'Akfadou (435 taxons), mais supérieur à celui obtenu par Bouchibane et al. (2017) pour les massifs forestiers de Kéfrida (332 taxons).

Les familles telles que les Asteraceae, les Fabaceae et les Poaceae sont bien représentées dans cette présente étude. Elles occupent la première position quant à leur richesse spécifique et générique et figurent parmi les plus grandes familles de la planète (Craven, 2009). La famille des orchidées occupe la quatrième place dans cette présente étude avec 21 taxons. Cette richesse en orchidées est due au fait que les massifs étudiés comportent un grand nombre de milieux préforestiers et ouverts (pelouses) qui offrent aux orchidées l'abri, l'humidité et la lumière, conditions écologiques favorables pour un bon nombre d'espèces d'orchidées. Ces résultats, que ce soit pour les genres ou les espèces, confirment les observations réalisées par Bouchibane et al. (2017) sur les massifs forestiers voisins de Kéfrida (Nord-est algérien). 


\subsection{Chorologie et endémisme}

Une étude phytogéographique constitue une base essentielle à toute tentative de conservation de la biodiversité (Quézel, 1999). Dans cette présente étude, l'élément méditerranéen est le mieux représenté avec un peu plus de $54 \%$ de la flore étudiée. Ce pourcentage est supérieur à ceux donné par Messaoudene et al. (2007) pour la forêt d'Akfadou avec $41 \%$ des espèces recensées et par Larbi (2014) pour la Cédraie de Tikjda (42,6\%), mais inférieur à celui indiqué par Bouchibane et al. (2017) pour les massifs montagneux de Kéfrida $(61,7 \%)$. Certains taxons de cet ensemble méditerranéen sont très rares sur le terrain, c'est le cas d'Acer monspessulanum L. subsp. martini (Jord) P. Four.; Erinacea anthyllis Link.; Kickxia lanigera (Desf.) Hand. - Mazz. ; Physospermum verticillatum (Waldst \& Kit.) vis ; Ruscus aculeatus L. et Scuttelaria columnae All. Ces taxons semblent être cantonnés exlusivement sur les versants nord du djebel Adrar Ou-Mellal dans un milieu forestier très humides à l'abri de la lumière.

La proportion des taxons appartenant au sous-élément oro-méditerranéen est important. Nous avons rencontré 9 taxons (tableau 2) sur les 29 signalés par Quézel \& Santa (1962-1963), soit $31,0 \%$ des oro-méditerranéen d'Algérie se retrouvent dans cette zone.

Selon Maire (1928), l'élément nordique s'est introduit en Afrique du Nord lors des périodes humides plus anciennes que le quaternaire à travers deux voies de migrations, une voie Ibérique (pont amalour-rifain) et une voie italienne (pont Sicilio-tunisien).

Le nombre de taxons appartenant à l'élément nordique (septentrional) est appréciable avec 101 espèces $(26,8 \%)$. Ce pourcentage est supérieur à ceux avancé par Gharzouli \& Djellouli (2005) pour le sud de la Kabylie des Babors (22\%), par Larbi (2014) pour la cedraie de Tikjda $(19,7 \%)$ et par Bouchibane et al. (2017) pour les massifs montagneux de Kéfrida (23,8\%). La plupart des espèces appartenant à cet élément nordique seraient installées, vraisemblablement, à la faveur d'un climat humide et rafraîchi correspondant aux phases glaciaires pléistocènes. Celles dont l'installation remonte aux périodes préglaciaires, notamment au Pliocène, ont pratiquement disparu, mis à part quelques vestiges (Quézel, 1983 \& 1995). Les modifications climatiques ultérieures ont entrainé la disparition de la plupart de ces espèces. Celles qui restent se limitent actuellement aux montagnes bien arrosées et aux zones humides (Maire, 1928 ; Quézel, 1995). Parmi les espèces de l'élément septentrional, Orchis purpurea Huds. subsp. purpurea. Cette orchidée rare figure dans un seul relevé effectué au massif Agouf à 970 m. d'altitude avec seulement 4 individus dans un maquis dominé par Calycotome spinosa (L.) Lam. et Ampelodesmos mauritanicus (Poir.) T. Durand. \& Schinz. Ce taxon n'a jamais été signalé auparavant en Kabylie des Babors.

Les massifs étudiés comptent 55 taxons endémiques régionaux, soit 14,6\% de la flore inventoriée, représentant ainsi 11,9\% des endémiques d'Algérie du nord estimée à 464 taxons par Dobignard \& Chatelain (2010-2013). Ce pourcentage d'endémisme (14,6\%) est supérieur à ceux donné par Rebbas (2014) pour le Parc national de Gouraya (5,6\%), par Bounar (2014) 
pour le Parc national de Taza (11,3\%) et par Bouchibane et al. (2017) pour les massifs montagneux de Kéfrida (11,1\%), mais comparable à celui indiqué par Gharzouli (2007) pour les massifs les plus méridionaux de la Kabylie des Babors (15,1\%). Le nombre d'espèces endémiques à répartition restreinte (endémiques strictes) est de $10(3 \%)$ pour les massifs de Kéfrida (Bouchibane et al. 2017), 14 (3,3\%) pour le Parc national de Taza (Bounar, 2014) et de $6(1,2 \%)$ pour le Parc national de Gouraya (Rebbas, 2014). Quant à nos massifs étudiés, ils comptent $14(3,7 \%)$ taxons endémiques à répartition restreinte. En région méditerranéenne, la richesse régionale en endémisme est due principalement à l'existence de nombreuses zones de persistance des espèces pendant les glaciations (Médail \& Diadema, 2009).

Trois espèces endémiques recensées sont propre à la Kabylie des Babors: Epimedium perralderanium Coss. ; Erodium battandieranum Rouy ; Moehringia stellarioides Coss.

Parmi les taxons endémiques que nous avons inventoriés, Cyclamen africanum Boiss. \& Reut.; Epimedium perralderanium Coss. ; Paeonia mascula (L.) Mill. subsp. atlantica (Cosson.) Greuter \& Burdet ; Phlomis bovei De Noé sont bien représentés sur le terrain. Ces taxons semblent trouver là des conditions favorable à leur épanouisement.

Certaines espèces endémiques signalées dans la bibliographie ancienne (Quézel, 1956; Quézel \& Santa, 1962-1963) pour la Kabylie des Babors, n’ont pas été observé, tels que, Dactylorhiza maculata subsp. Battandieri (Raynaud) Baumann \& Kunkele ; Cyclamen repandum Sm.; Silene reverchoni Batt. Ces trois taxons sont aussi absents des inventaires floristiques effectués récemment dans la région de la Kabylie des Babors (Gharzouli \& Djellouli, 2005 ; Gharzouli, 2007). Il est probable que les changements climatiques de ces dernières années ont des effets néfastes sur ces taxons devenus rarissime, ils seraient à rechercher dans les endoits d'altitudes les plus inaccessibles à l'homme et à son bétail.

Les espèces appartenant à l'ensemble à large répartition correspondent à des éléments de transition entre l'ensemble méditerranéen et les ensembles chorologiques voisins (Bounar, 2014). Les espèces de cet ensemble sont au nombre de 15 , soit $4,0 \%$ de la flore analysée. Ce pourcentage est comparable à ceux avancé par Gharzouli \& Djellouli (2005) pour le sud de la Kabylie des Babors (3\%) et par Bouchibane et al. (2017) pour les massifs montagneux de Kéfrida (3,3\%). Parmi elles, les cosmopolites et les subcosmopolites qui sont faiblement représentés avec seulement 10 espèces. Ce faible taux s'explique par le fait que l'échantillonnage a concerné uniquement les forêts d'altitude où la pression anthropozoïque, particulièrement l'activité agricole, est moins forte que sur les bas de versants (Gharzouli, 2007). Les espèces tropicales sont très faiblement représentées dans cette présente étude avec $1,3 \%$ de la flore étudiée.

\subsection{Types biologiques}

Les massifs étudiés sont dominés par les hémicryptophytes $(43,8 \%)$. Ce pourcentage des hémicryptophytes est comparable à celui obtenu par Larbi (2014) pour la Cédraie de Tikjda (45\%). La dominance des hémicryptophytes peut être expliquée par la pluviosité et le froid 
qui règne surtout en hiver (Floret et al., 1990), mais aussi par les hautes altitudes et la richesse du sol en matière organique (Kazi Tani et al., 2010). Il semblerait que les précipitations et la couverture végétale favorisent le développement des hémicryptophytes (Bouchibane et al., 2017) ; ajoutons à cela l'importance des mycorhizes dans le sol (Whigham, 2004).

Les thérophytes occupent la deuxième place avec 86 espèces $(22,8 \%)$. Ce pourcentage est inférieur à ceux indiqué par Gharzouli (2007) pour les massifs du sud de la Kabylie des Babors (29\%) et par Rebbas (2014) pour le Parc National de Gouraya (37,3\%), mais comparable à ceux avancé par Bouchibane et al. (2017) pour les massifs montagneux de Kéfrida (22,5\%) et par Larbi (2014) pour la Cédraie de Tikjda (23\%). Le surpâturage et les incendies répétés observés sur le terrain surtout durant la saison estivale conduisant à la dégradation de la couverture végétale, ce qui favorise l'apparition des thérophytes. Les géophytes sont représentés par 51 taxons (13,5\%). Ils ne présentent pas une tendance particulière, mais ils semblent avoir une préférence pour les milieux ouverts, notamment les matorrals et les pelouses de montagnes.

Les phanérophytes sont représentés par 48 espèces (12,7\%). Ces plantes, bien plus résistantes que les autres types biologiques, sont constituées essentiellement d'arbustes perennes, des arbres sclérophylles et des lianes. Verlaque et al. (2001) soulignent que la position priviligiée des phanérophytes repose sur une stratégie optimale de compétition : grande taille, longivité, bon semencier, en général allogamie. Malgré leur faible diversité spécifique, elles dominent parfois par leur recouvrement et jouent de ce fait un rôle déterminant dans la mise en place d'un cortège floristique spécifique aux milieux forestiers (Lecompte-Barbet, 1975).

Les chaméphytes se présentent en faibles proportions avec seulement 27 taxons, soit 7,2\% de la flore étudiée. Ce pourcentage est comparable à celui avancé par Messaoudene et al. (2007) pour la forêt d'Akfadou (8\%). Les chaméphytes seraient bien adaptées au phénomène d'aridification des sols, car ils peuvent développer diverses formes d'adaptation à la sécheresse, se traduisant par la réduction de la surface foliaire et par le développement d'un puissant système racinaire (Floret et al., 1990). Selon Kadi-Hanafi (2003), le nombre de chaméphytes progresse avec l'aridité et l'ouverture du milieu.

\subsection{Rareté et valeur patrimoniale}

Les espèces rares sont considérées comme ayant une faible abondance et/ou une aire de répartition restreinte (Rebbas, 2014). La flore analysée compte 114 (30,2\%) espèces rares s.l. Ce pourcentage $(30,2 \%)$ est supérieur à celui donné par Rebbas (2014) pour le Parc National de Gouraya (11,2\%), mais comparable à ceux avancé par Bounar (2014) pour le Parc national de Taza (27\%) et par Bouchibane et al. (2017) pour les massifs montagneux de Kéfrida $(32,2 \%)$. Les espèces recensées, comme Epimedium perralderianum Coss. (10\% des relevés); Erodium battandieranum Rouy (5,6\% des relevés) ; Lonicera kabylica Rehder (3,3\% des relevés) ; Moehringia stellarioides Coss. (2,2\% des relevés) ; Phlomis bovei De Noé (14,4\% des relevés); Pimpinella battandieri Chabert $(3,3 \%$ des relevés) ; Sedum multiceps Coss. \& Durieu (6,7\% des relevés) ; Selinopsis montana Batt. (5,6\% des relevés) ; 
Teucrium atratum Pomel (2,2\% des relevés) et Thymus dreatensis Batt. (4,4\% des relevés) sont à la fois endémiques et rares. Ces dix taxons figurent aussi sur la liste rouge de l'UICN 1997 (Walter \& Gillett, 1998) dont un avec un statut en danger (Epimedium perralderianum Coss.) et l'autre vulnérable (Thymus dreatensis Batt.).

Les massifs étudiés comptent onze taxons figurant sur la liste des espèces déterminantes (trigger species) pour l'Algérie (Yahi et al., 2012) à savoir : Dactylorhiza munbyana (Boiss. \& Reut.) Aver.; Epimedium perralderianum Coss. ; Erodium battandieranum Rouy ; Heracleum sphondylium L. subsp. algeriense (Batt. \& Trab.) ; Lonicera kabylica (Batt.) Rehder; Moehringia stellarioides Coss. ; Paeonia mascula (L.) Mill. subsp. atlantica (Coss.) Greuter \& Burdet; Phlomis bovei De Noé ; Scuttelaria columnae All.; Sedum multiceps Coss. \& Durieu ; Silene choulettii Coss.

Ce nombre d'espèces déterminantes (11) est comparable à celui obtenu par Bouchibane et al. (2017) pour la forêt de Kéfrida avec 12 espèces, mais supérieur à celui donné par Miara et al. (2017) pour la flore de l'Atlas tellien occidental de Tiaret (Nord-Ouest algérien) avec 4 espèces. Pour Viburnum lantana L. le massif d'Adrar Ou-Mellal, en constitue une nouvelle station, la plus proche connue, étant celle du mont Babor.

Certaines espèces recensées comme Limodorum arbortivum (L). Sw. ; Potentilla caulescens L. ; Lysimachia cousiniana Coss. \& Durieu ne figurent guère dans les inventaires réalisés récemment (Gharzouli \& Djellouli, 2005 ; Gharzouli, 2007 ; Bouchibane et al., 2017) au niveau des autres massifs de la région des Babors (Tababort, Babor, Sidi-Djaber et Adrar N'fad). Il semblerait que le massif d'Adrar Ou-Mellal, soit leur unique station dans la région des Babors.

Les documents inhérents aux espèces rares et menacées (Walter \& Gillet, 1998 ; D. E 2012) nous semblent incomplets, un inventaire le plus exhaustif possible de la flore algérienne reste à faire. Des espèces très rares, localisées uniquement dans les massifs étudiés et zones limitrophes, ne figurent pas sur la liste des espèces protégées (Tableau 5). Nos observations sur le terrain nous amènent à proposer une liste de 8 taxons à faire bénéficier de mesures de protection: Acer monspessulanum L. subsp. martini (Jord.) P. Fourn.; Heracleum sphondylium L. subsp. algeriense (Batt. \& Trab.) Dobignard; Physospermum verticillatum (Waldst. \& Kit.) Vis. ; Plagius maghrebinus Vogt \& Greuter; Scutellaria columnae All. ; Silene choulettii Coss. ; Stellaria holostea L. ; Veronica montana L.

\subsection{Menaces et mesures de conservation}

La forêt des massifs étudiés révèle une dégradation préoccupante. En effet, les activités anthropiques, surtout les incendies de forêts, le tourisme de masse, le surpâturage et l'exploitation anarchique des espèces connues pour leurs vertus thérapeutiques (Ajuga iva (L.) Schreber ; Origanum vulgare subsp. glandulosum (Desf.) Letswaart) portent un sérieux préjudice à cette richesse floristique. Une autre situation inquiétante a été obersvée au niveau du massif d'Adrar Ou-Mellal $(1773 \mathrm{~m})$ qui est le dépérissement que connaissent certains 
peuplements de cèdres. D'après Sarmoum et al. (2019), les précipitations moins importantes, les températures de plus en plus élévées, et ainsi que l'âge avancé des arbres sont les principales causes de ce phénomène.

Pour faire face à cette problématique et garder l'intégrité écologique de cette flore, une stratégie intégrée de conservation de la biodiversité doit être mise en place. Cette stratégie doit se focaliser en premier lieu sur les essences forestières qui par leur unicité constituent la charpente essentielle de cet écosystème naturel. Il s'agit en particulier du Cèdre (Cedrus atlantica (Endl.) Carrière), du Chêne Zéen (Quercus canariensis Willd.) et du Chêne liège (Quercus suber L.). En effet, ces arbres constituent les principales formations forestières des massifs étudiés et hébergent dans leurs cortèges floristiques plusieurs espèces endémiques ou/et rares comme Epimedium perralderianum Coss.; Erodium battandieranum Rouy; Moehringia stellarioides Coss.; Physospermum verticillatum (Waldst \& Kit.) vis ; Thymus dreatensis Batt. Ces espèces trouvent ici leur unique station en Algérie, auxquelles une attention toute particulière doit être accordée.

\section{Conclusion}

Les conditions climatiques qui prédominent en Kabylie des Babors en général et au sein des massifs étudiés en particulier, et le relief très accidenté ont permis le maintient d'une flore riche et diversifiée avec près de 400 taxons inventoriés dans les massifs analysés (Agouf et Adrar Ou-Mellal). Cette étude a permis la découverte de nouvelles stations pour Orchis purpurea Huds. subsp. purpurea et Viburnum lantana L. Cet inventaire nous a permis aussi de renforcer et compléter les connaissances sur la flore locale et d'apporter plus d'information sur le plan de la distribution géographique de certains taxons. En outre, les deux massifs étudiés comportent six taxons remarquables qui ne sont pas représentés sur les autres massifs du secteur des Babors.

L'analyse du spectre chorologique brut montre une dominance de l'élément méditerranéen $(54,6 \%)$, suivie par l'ensemble septentrional $(26,8 \%)$. L'ensemble endémique régional est assez bien représenté avec 14,6\% des espèces répertoriées. Les types biologiques des massifs étudiés sont dominés par les hémicryptophytes $(43,8 \%)$. De plus, ces massifs comptent onze taxons figurant sur la liste des espèces déterminantes pour l'Algérie.

Le classement des massifs étudiés (Adrar Ou-Mellal et Agouf) ainsi que tous les autres massifs montagneux de la Kabylie des Babors en Parc naturel régional est nécessaire pour assurer son équilibre biologique et pour sauvegarder son patrimoine floristique et forestier, cela est justifié par le nombre assez conséquant d'espèces endémiques, rares, menacées et protégées et permettra de préserver les différents habitats des massifs étudiés. Il favorisera aussi la mise en œuvre d'une politique de conservation qui prendra en compte les préoccupations socio-économiques des riverains. 
Bulletin de la Société Royale des Sciences de Liège, Vol. : 91, articles, 2021, p. 317 - 360

\section{Remerciements}

Nous tenons à remercier vivement M. REBBAS Khellaf, enseignant à l'université de M'sila (Algérie), et M. VELA Errol, enseignant à l'université de Montpellier (France) pour leur aide dans la détermination des espèces.

\section{Réferences bibliographiques}

Amirouche R. \& Misset M-Th., 2009. Flore spontanée d'Algérie : Différenciation écogéographique des espèces et polyploidie. Cahiers d'Agriculture 18 : 474-480.

APD 2021. African Plant Database (version 3.4.0). Conservatoire et Jardin botaniques de la Ville de Genève and South African National Biodiversity Institute, Pretoria, "Retrieved 3th March 2021", from http://africanplantdatabase.ch

Battandier J.A., 1888-1890. Flore de l'Algérie : Dicotylédones. A. Jourdan (ed.), Alger. 855p.

Battandier J.A. \& Trabut L., 1895. Flore d'Algérie, contenant la description de toutes les plantes signalées jusqu'à ce jour comme spontanées en Algérie et catalogue des plantes du Maroc: Monocotylédones. A. Jourdan (ed.). Alger.

Benhouhou S., Yahi N., Véla E., 2018. Algeria. In: Conserving wild plants in the South and East Mediterranean region (chapter 3 "Key Biodiversity Areas (KBAs) for plants in the Mediterranean region”) (eds Valderrábano, M., Gil, T., Heywood, V. \& Montmollin de, B.). Gland, pp. 53-60.

Benoit G. \& Comeau A., 2005. Méditerranée, les perspectives du plan bleu pour l'environnement et le développement. Plan Bleu et Aube. Eds, 432p.

Bouchibane M., Véla E., Bougaham A.F., Zemouri M., Mazouz A. \& Sahnoune M., 2017. Étude phytogéographique des massifs forestiers de Kéfrida, un secteur méconnu de la zone importante pour les plantes des Babors (Nord-est algérien). Revue d'Ecologie (terre et vie), vol. 72 (4) : 374-386. https://core.ac.uk/download/pdf/199284721.pdf

Bougaham A.F., Rebbas K. \& Véla E., 2020. Découverte d'Epipactis microphylla (Orchidaceae) au djebel Babor (nord-est de l'Algérie), orchidée nouvelle pour l'Afrique du Nord. Flora Mediterranra, 30 : 261-271.

Bounar R., 2014. Étude des potentialités biologiques, cartographie et aménagement de la chaine des Babors dans la démarche du développement durable. Thèse de Doctorat, Université de Sétif (Algérie), 118p.

Chelli-Tabti D., Markhouf S., Derradji S., Hamitouche S., Bouchareb A. \& Bougaham A.F., 2020. New data on the distribution area of the Atlas foxglove Digitalis atlantica (Pomel). 
Bulletin de la Société Royale des Sciences de Liège, Vol. : 91, articles, 2021, p. 317 - 360

Ecologia mediterranea. Vol 46 (2): 41-47. https://ecologia-mediterranea.univ-avignon.fr/wpcontent/uploads/sites/25/2020/10/EM-46-1-corpus-web-BR.pdf\#page=43

Craven P., 2009. Phytogeographic study of the Kaokoveld centre of endemism. PhD. Dissertation, University of Sttellenbosch, 233p.

Daki M., 2004. Étude de Faisabilité d'un Plan d'Aménagement Côtier en Méditerranée Marocaine, Rapport final du Département de l'environnement. Ministère de L'aménagement du Territoire, de l'Eau et de l'Environnement, Rabat. Maroc, 6-113.

Dobignard A. \& Chatelain C., 2010 - 2013. Index synonymique de la flore d'Afrique du Nord. Ed. Conservatoire et Jardin Botanique, Genève.

Duplan L., 1952. La région de Bougie. Publ. XIX Congr. Geol. Int. Monog. Rég. $1^{\mathrm{er}}$ Série Algérie, 17. 40p.

Emberger L., 1955. Une classification biogéographique des climats. Nat. Monsp., Série Bot, 7: 3-42.

Euro+Med (2006-2021): Euro+Med PlantBase - the information resource for EuroMediterranean plant diversity. Published on the Internet: http://ww2.bgbm.org/EuroPlusMed/ [accessed $3^{\text {th }}$ March 2021].

Fennane M., Ibn Tattou M. \& El Oualidi J., 2014. Flore pratique du Maroc : Manuel de détermination des plantes vasculaires.Vol. I-III. Institut Scientifique, Université Mohammed V - Agdal, Rabat.

Floret C.H., Galan M.J., Le floc H., Orshan G., \& Romane F., 1990. Growth forms and phenomorphology traits along an environmental gradient: tools for studying Vegetation. Journal of Vegetation Science, 1: 71-80.

Gharzouli R., 2007. Flore et végétation de la Kabylie des Babors. Etude floristique et phytosociologique des groupements forestiers et post-forestiers des djebels Takoucht, Adrar Ou-Melal, Tababort et Babor. Thèse de Doctorat, Université de Sétif (Algérie), 356p.

Gharzouli R. \& Djellouli Y., 2005. Diversité floristique de la Kabylie des Babors (Algérie). Sécheresse, 16 (3) : 217-223.

https://www.botaniquealgerie.dz/sites/default/files/pdf/publications/04-2018/diversitefloristique-de-la-kabylie-des-babors-algerie.pdf

Gounot M., 1969. Méthodes d'étude quantitatives de la végétation. Éd. Masson, Vol 1, 314p. 
Bulletin de la Société Royale des Sciences de Liège, Vol. : 91, articles, 2021, p. 317 - 360

J.O.R.A., 2012. Décret exécutif du 18 Janvier 2012, complétant la liste des espèces végétales non cultivées et protégées. Journal officiel de la République Algérienne, n 3-12/12 du 18-012012, 27p.

Jeanmonod D. \& Gamisans J., 2007. Flora corsica. Edit. Edisud, Aix-en- Provence, 920p.

Kadi-Hanifi H., 2003. Diversité biologique et phtytogéographique des formations à Stipa tenacissima L. de l'Algérie. Sécheresse, 14 (3) : 169-179.

Kazi Tani C., Lebourgeois T. \& Munoz F., 2010. Aspects floristiques de la flore des champs du domaine phytogéographique oranais (Nord-Ouest algérien) et persistance d'espèces rares et endémiques. Fl. Medit., 20: 5-22. https://www.herbmedit.org/flora/20-029.pdf

Larbi N., 2014. Analyse de la diversité floristique et de la phytodynamique de la série de végétation à Cedrus atlantica Manetti au Djurdjura centro-méridional (secteur de Tikjda). Mémoire de Magister, Université de Tizi-Ouzou, 106p.

Lecompte-Barbet O., 1975. Introduction à une étude de l'endémisme végétal au Maroc. Pp 1546 in: Étude de certains milieux au Maroc et de leur évolution récente. Travaux de la R.C.P. 249, CNRS., Paris.

Maire R., 1926. Carte phytogéographique de l'Algérie et de la Tunisie - 1/1 500 000, Gouvernement Général d'Alger, Service Cartographie., Alger, 78p.

Maire R., 1928. Origine de la flore des montagnes de l'Afrique du Nord. Mém. Soc. Biog., 2 : 187-194.

Maire R., 1952-1987. Flore de l'Afrique du Nord (Maroc, Algérie, Tunisie, Tripolitaine, Cyrénaïque, Sahara). Vol. I-XVI. Lechevalier, Paris.

Médail F. \& Diadema K., 2009. Glacial refugia influence plant diversity patterns in the Mediterranean Basin. Journal of biogeography, 36 (7). 1333-1345. https://doi.org/10.1111/j.1365-2699.2008.02051.x

Médail F. \& Quézel P., 1997. Hotspot analysis for conservation of plants biodiversity in the Méditerranéan Basin. Ann. Miss. Bot. Gard, 84: 112-127. https://doi.org/10.2307/2399957

Meddour R., 2010. Bioclimatologie, phytogéographie et phytosociologie en Algérie. Exemple des groupements forestiers et préforestiers de la Kabylie Djurdjureenne. Thèse de doctorat en foresterie. Université de Tizi-Ouzou, 397p.

Messaoudene M., Laribi M. \& Derridj A., 2007. Étude de la diversité floristique de la forêt de l'Akfadou (Algérie). Bois et Forêts des Tropiques. 291 (1) : 75-81.

https://revues.cirad.fr/index.php/BFT/article/view/20359 
Bulletin de la Société Royale des Sciences de Liège, Vol. : 91, articles, 2021, p. 317 - 360

Miara M.D., Ait Hammou M., Rebbas K., 2017. Flore endémique, rare et menacées de l'Atlas tellien occidental de Tiaret (Algérie). Acta Botanica Malacitana, 42 (2), General, 269-283. DOI : https://doi.org/10.24310/abm.v42i2.3590 https://revistas.uma.es/index.php/abm/article/view/3590

Pignatti S., 1982. Flora d'Italia. Edagricole, Bologna, Vol. I-III, 790, 732 et 780p.

Quézel P., 1956. Contribution à l'étude des forêts de chênes à feuilles caduques d'Algérie Mém. Soc. Hist. Nat. Afrique du Nord. Nouv. Série. 1, Alger, 57p.

Quézel P., 1978. Analysis of the flora of Mediterranean and Saharan Africa. Ann. Missouri Bot. Garden, 65: 479-537.

Quézel P., 1983. Flore et végétation actuelles de l'Afrique du Nord, leurs significations en fonction de l'origine, de l'évolution et des migrations des flores et structures de végétation passées. Bothalia, 14: 411-416.

Quézel P., 1985. Definition of the Mediterranean region and the origin of its flora, in: C.

Gomez-Campo (Ed.), Plant Conservation in the Mediterranean Area, Dordrecht, the Netherlands, pp. 9-24.

Quézel P., 1995. La flore du bassin méditerranéen : origine, mise en place, endémisme. Ecologia mediterranea, $21: 19-39$.

Quézel P., 1999. Les grandes structures de végétations en région méditerranéenne : facteurs déterminants dans la mise en place post-glaciaire. Geobios, 32(1) : 19-32.

Quézel P. \& Médail F., 2003. Écologie et biogéographie des forêts du Bassin méditerranéen. Elsevier, Paris, 571p.

Quézel P. \& Santa S., 1962-1963. Nouvelle flore de l'Algérie et des régions désertiques méridionales. 2 volumes, CNRS, Paris, 1170p.

Raunkiaer C., 1934. The life form of plants and statistical plant geography. Collected papers, Clarendon Press, Oxford, 632p.

Rebbas K., 2014. Développement durable au sein des aires protégées algériennes, cas du Parc National de Gouraya et des sites d'intérêt biologique et écologique de la région de Bejaia. Thèse de Doctorat, Université de Sétif (Algérie), 180p.

Rebbas K., Vela E., Bougaham A.F., Belharrat A., De Belaire G. \& Prelli R., 2019. Découverte de Christella dentata (Thelypteridaceae) en Algérie. Fl. Medit. 29, 55-66. 
Bulletin de la Société Royale des Sciences de Liège, Vol. : 91, articles, 2021, p. 317 - 360

https://doi.org/10.7320/FlMedit29.055.

Sarmoum M., Navarro-Cerillo R. \& Guibal F., 2019. Bilan actuel et rétrospéctif du dépérissement du cèdre de l'Atlas dans le Parc national de Theniet El Had (Algérie). Bois et Forêts des Tropiques. Vol 342 (4) : 29- 40. https://doi.org/10.19182/bft2019.342.a31636

Seltzer P., 1946. Le climat de l'Algérie. Inst. Météor. et de Phys. du Globe. Univ. Alger, 219 p.

Véla E. 2018. De l'inventaire de la biodiversité aux priorités de conservation dans le hotspot du bassin méditerranéen : peut-on combler les déficits de connaissance? Diplôme d'Habilitation à Diriger les Recherches, Université Montpellier, 64p.

Véla E. \& Benhouhou S., 2007. Évaluation d'un nouveau point chaud de biodiversité végétale dans le bassin méditerranéen (Afrique du nord). C.R. Biologies, 330 : 589-605.

DOI : $\underline{10.1016 / \text { j.crvi.2007.04.006 }}$

Vérlaque R., Médail F. \& Aboucaya A., 2001. Valeur prédictive des types biologiques pour la conservation de la flore méditérranéenne. Life Sci. 324 : 1157-1165.

Walter K.S. \& Gillett H.J., 1998. 1997 IUCN red list of threateaned plants. Compiled by the World Conservation Monitoring Centre. IUCN - the World Conservation Union. Gland, Switzerland and Cambridge. UK. Ixiv $+862 p$.

Whigham D.F., 2004. Ecology of woodland herbs in temperate deciduous forests. Ann. Rev. Ecol. Evol. Syst., 35: 583-617. https://doi.org/10.1146/annurev.ecolsys.35.021103.105708

Yahi N., Véla E., Benhouhou S., De Bélair G. \& Gharzouli R., 2012. Identifying important plants area (Key Biodiversity Area for Plants) in northern Algeria. Journal of Threatened Taxa, 4: 2753-2765. DOI : https://10.11609/JoTT.o2998.2753-65 
Bulletin de la Société Royale des Sciences de Liège, Vol. : 91, articles, 2021, p. 317 - 360

Annexe 1 : Liste des espèces inventoriées dans les massifs étudiés, leur biogéographie, leur type biologique et leur appréciation d'abondance d'après les données bibliographiques de Quézel \& Santa (1962-1963). AR : Assez rare, R : Rare, RR : Très rare. AC : Assez commun. C : Commun. CC : Très Commun. CCC : Particulièrement répandu. Hemi : Hémicryptophytes. Geo : Géophytes. Cham : Chaméphytes. Ther: Thérophytes. Phan: Phanérophytes. End: Endémique. Med: Méditerranéen. Statut : appréciation d'abondance.

\begin{tabular}{|c|c|c|c|}
\hline $\begin{array}{c}\text { Nom des plantes selon Dobignard \& } \\
\text { Chatelain (2010-2013) }\end{array}$ & Biogéographie & Type biologique & Statut \\
\hline \multicolumn{4}{|l|}{ Asteraceae } \\
\hline Anacyclus perythrum (L.) Link. & Eurymed & Hemi & $\mathrm{C}$ \\
\hline Andryala integrefolia $\mathrm{L}$. & Euryméd & Ther & $\mathrm{CC}$ \\
\hline $\begin{array}{l}\text { Anthemis pedunculata Desf. subsp. } \\
\text { pedunculata }\end{array}$ & Stenomed & Hemi & AR \\
\hline Anthemis punctata Vahl. & Oro-Euras & Hemi & $\mathrm{R}$ \\
\hline Atractylis cancellata $\mathrm{L}$. & Stenomed & Ther & $\mathrm{CC}$ \\
\hline Bellis annua.L .subsp. annua & Sténoméd & Ther & $\mathrm{CCC}$ \\
\hline Bellis sylvestris Cirillo, sensu lato & Sténoméd & Hémi & $\mathrm{CCC}$ \\
\hline Calendula suffruticosa Vahl., sensu lato & Med & Hemi & $\mathrm{CC}$ \\
\hline Calendula arvensis L. var. bicolor & Med & Hemi & $\mathrm{CCC}$ \\
\hline Carthamus multifidus Desf. & End N.Afr. & Cham & $\mathrm{CCC}$ \\
\hline Carduus macrocephalus Desf. & Sténoméd & Hemi & $\mathrm{CC}$ \\
\hline Carduus pychnocephalus L. & Euras & Hemi & $\mathrm{CC}$ \\
\hline Catananche montana Coss. & End Alg+Mar & Hemi & AR \\
\hline Catananche caerulea $\mathrm{L}$. & W. Med & Hemi & $\mathrm{C}$ \\
\hline Centaurea sphaerocephala $\mathrm{L}$. & Stenomed & Hemi & $\mathrm{CCC}$ \\
\hline Centaurea pullata $\mathrm{L}$. & Euryméd & Ther & $\mathrm{CC}$ \\
\hline Centaurea pubescensWilld. & Stenomed & Cham & $\mathrm{CC}$ \\
\hline Cichorium intybus $\mathrm{L}$. & Cosmopolite & Hemi & $\mathrm{CC}$ \\
\hline Crepis vesicaria L. subsp. taraxacifolia & Atlantique-Med & Hemi & $\mathrm{AC}$ \\
\hline
\end{tabular}


Bulletin de la Société Royale des Sciences de Liège, Vol. : 91, articles, 2021, p. 317 - 360

\begin{tabular}{|c|c|c|c|}
\hline (Thuill.) Thell. & & & \\
\hline Dittrichia viscosa (L.) Greuter & Euryméd & Hemi & $\mathrm{CC}$ \\
\hline $\begin{array}{l}\text { Doronicum plantaginum subsp. atlanticum } \\
\text { (Chabert.) Rouy. }\end{array}$ & End N.Afr. & Hemi & $\mathrm{AC}$ \\
\hline Echinops bovei Boiss. & Eurymed & Cham & $\mathrm{CC}$ \\
\hline Filago pyramidata $\mathrm{L}$. & Eurymed & Ther & $\mathrm{CC}$ \\
\hline Galactites tomentosus Moench. & Sténoméd & Hémi & $\mathrm{CCC}$ \\
\hline Helichrysum pendulum (C. Presl.) C. Presl & Stenomed & Cham & $\mathrm{CCC}$ \\
\hline Hieracium pseudopilosella (Ten.) Sojak & End N.Afr. & Hemi & $\mathrm{CC}$ \\
\hline Hyoseris radiata $\mathrm{L}$. & Stenomed & Hemi & $\mathrm{CC}$ \\
\hline Hypochoeris radicata $\mathrm{L}$. & Stenomed & Ther & $\mathrm{CCC}$ \\
\hline Hypochoeris laevigata $\mathrm{L}$. & SW-Med & Cham & $\mathrm{CC}$ \\
\hline Leontodon saxatilis Lam. & W-Med & Ther & $\mathrm{CC}$ \\
\hline Mantisalca salmantica (L.) Bricq \& Carvill. & Stenomed & Hemi & $\mathrm{CC}$ \\
\hline Pallenis spinosa L. Cass. subsp. spinosa & Eurymed & Hemi & $\mathrm{CC}$ \\
\hline $\begin{array}{l}\text { Pentanema montanum (L.) D. Gut. } \\
\text { Larr.Santos-Vicente \& al. }\end{array}$ & Atlantique & Hémi & $\mathrm{AC}$ \\
\hline Petasites pyrenaicus (L.) G. Lopez. & C. Med & Geo & $\mathrm{AC}$ \\
\hline Phagnalon sordidum (L.) Rchb. & W. Med & Hemi & $\mathrm{AC}$ \\
\hline Plagius maghrebinus Vogt \& Greuter. & End N.Afr. & Cham & $\mathrm{C}$ \\
\hline Pulicaria odora (L.) Rchb. & Euryméd & Hémi & $\mathrm{CC}$ \\
\hline Reichardia picroides (L.) Roth. & Stenomed & Hemi & $\mathrm{CCC}$ \\
\hline Santolina pectinata Lag. & SW-Med & Cham & $\mathrm{R}$ \\
\hline Senecio perralderianus Coss \& Dur. & End Alg & Hemi & $\mathrm{AC}$ \\
\hline Solidago virgaurea L., subsp. virgaurea & Boreal & Hemi & $\mathrm{AR}$ \\
\hline Taraxacum inaequilobum Pomel & End N.Afr. & Hemi & $\mathrm{AR}$ \\
\hline Tussilago farfara $\mathrm{L}$. & Paleotemp. & Geo & $\mathrm{R}$ \\
\hline $\begin{array}{l}\text { Urospermum dalechampsii (L.) F. W. } \\
\text { Schmidt. }\end{array}$ & Eurymed & Hemi & $\mathrm{CC}$ \\
\hline
\end{tabular}


Bulletin de la Société Royale des Sciences de Liège, Vol. : 91, articles, 2021, p. 317 - 360

\begin{tabular}{|c|c|c|c|}
\hline Fabaceae & & & \\
\hline Anthyllis montana $\mathrm{L}$. & Oro-Europ & Hemi & $\mathrm{R}$ \\
\hline $\begin{array}{l}\text { Anthyllis vulneraria L. subsp. maura (Beck.) } \\
\text { Maire }\end{array}$ & Eurymed & Hemi & $\mathrm{CC}$ \\
\hline Argyrolobium zanonii (Turra) P. W. Ball & Eurymed & Phan & $\mathrm{C}$ \\
\hline $\begin{array}{l}\text { Astragalus monspessulanus L. subsp. } \\
\text { gypsophilus Rouy }\end{array}$ & W. Med & Hemi & $\mathrm{AC}$ \\
\hline $\begin{array}{l}\text { Bituminaria bituminosa (L.) C .H. Stirt., sensu } \\
\text { lato }\end{array}$ & Stenomed & Hemi & $\mathrm{C}$ \\
\hline Calicotome spinosa (L.) Lam. & Sténoméd & Phan & $\mathrm{CC}$ \\
\hline Ceratonia siliqua $\mathrm{L}$. & Stenomed & Phan & $\mathrm{C}$ \\
\hline $\begin{array}{l}\text { Coronilla valentina L. subsp. speciosa } \\
\text { (Uhrova) Greuter \& Bur }\end{array}$ & End Alg & Cham & $\mathrm{AC}$ \\
\hline $\begin{array}{l}\text { Coronilla minima L., subsp. lotoides (W.D.J. } \\
\text { Koch) Nyman }\end{array}$ & Eurymed & Cham & $\mathrm{AC}$ \\
\hline Coronilla scoprpoides (L.) W. D. J. Koch. & Eurymed & Ther & $\mathrm{C}$ \\
\hline Cytisus villosus Pourr. & Stenomed & Phan & $\mathrm{C}$ \\
\hline Erinacea anthyllis Link. & Oro-Med & Phan & AR \\
\hline Genista triscupidata Desf. & Eurymed & Phan & $\mathrm{CC}$ \\
\hline Hippocrepis atlantica Ball & End Alg-Mar & Hemi & $\mathrm{C}$ \\
\hline Hippocrepis biflora Spreng. & Eurymed & Hemi & $\mathrm{C}$ \\
\hline Lathyrus latifolius L. & C. Europe & Hemi & $\mathrm{CC}$ \\
\hline Lathyrus articulatus L. & Euryméd & Ther & $\mathrm{CC}$ \\
\hline Lotus ornithopodioides L. & Stenomed & Ther & $\mathrm{C}$ \\
\hline Lotus creticus L. & Stenomed & Ther & $\mathrm{AC}$ \\
\hline Medicago truncatula Gaertn. & Stenomed & Ther & $\mathrm{C}$ \\
\hline Medicago polymorpha $\mathrm{L}$. & Eurymed & Ther & $\mathrm{C}$ \\
\hline Medicago orbicularis (L.) Bartal. & Eurymed & Ther & $\mathrm{AC}$ \\
\hline Medicago murex Willd. & Stenomed & Ther & $\mathrm{C}$ \\
\hline Medicago rigidula (L.) All. & Eurymed & Hemi & $\mathrm{R}$ \\
\hline
\end{tabular}


Bulletin de la Société Royale des Sciences de Liège, Vol. : 91, articles, 2021, p. 317 - 360

\begin{tabular}{|c|c|c|c|}
\hline Onobrychis caput-galli (L.) Lam. & Stenomed & Ther & $\mathrm{CC}$ \\
\hline $\begin{array}{l}\text { Ononis viscosa L. subsp. breviflora (DC.) } \\
\text { Nyman. }\end{array}$ & Eurymed & Ther & $\mathrm{C}$ \\
\hline Ononis alopecuroides L. & Stenomed & Ther & AR \\
\hline $\begin{array}{l}\text { Scorpiurus muricatus L. subsp. sulcatus (L.) } \\
\text { Thell. }\end{array}$ & Stenomed & Ther & $\mathrm{C}$ \\
\hline Trigonella monspeliaca $\mathrm{L}$. & Stenomed & Ther & $\mathrm{AC}$ \\
\hline Tripholium ochroleucon Huds. & Euras & Hemi & AR \\
\hline Trifolium pratense $\mathrm{L}$. & Euro-sibérie & Hemi & AR \\
\hline Trifolium campestre Schreb. & Paleotemp & Ther & $\mathrm{CC}$ \\
\hline Tripholium stellatum L. & Euryméd & Ther & $\mathrm{CC}$ \\
\hline Vicia lathyroides L. & Stenomed & Ther & $\mathrm{AR}$ \\
\hline Vicia onobrychoides L. & Med-Mont & Hemi & $\mathrm{AC}$ \\
\hline \multicolumn{4}{|l|}{ Poaceae } \\
\hline Aegilops ventricosa Tausch. & Stenomed & Ther & $\mathrm{C}$ \\
\hline Aira tenorii Guss. & Stenomed & Ther & $\mathrm{AC}$ \\
\hline $\begin{array}{l}\text { Ampelodesmos mauritanicus (Poir.) T. } \\
\text { Durand. \& Schinz. }\end{array}$ & W. Eurymed & Hemi & $\mathrm{CC}$ \\
\hline Anisantha sterilis (L.) Nevski. & Méd-Tour & Ther & $\mathrm{C}$ \\
\hline Anisantha tectorum (L.) Nevski. & Paleotemp & Ther & $\mathrm{C}$ \\
\hline Anisantha rubens L. Nevski & Paléosubtropical & Ther & $\mathrm{C}$ \\
\hline Anisantha madritensis (L.) Nevski. & Eurymed & Ther & $\mathrm{CC}$ \\
\hline Anthoxanthum odoratum L. & Circumboréal & Hemi & $\mathrm{AC}$ \\
\hline Avena sterilis $\mathrm{L}$. & Med & Ther & $\mathrm{C}$ \\
\hline $\begin{array}{l}\text { Brachypodium phoenicoides (L.) P.Beauv. ex } \\
\text { Roem. \& Schult. }\end{array}$ & Stenomed & Hemi & $\mathrm{C}$ \\
\hline $\begin{array}{l}\text { Brachypodium sylvaticum (Huds). P.Beauv. } \\
\text { subsp. sylvaticum. }\end{array}$ & Paleotemp & Hemi & $\mathrm{CC}$ \\
\hline Briza maxima $\mathrm{L}$. & Sub-tropical & Ther & $\mathrm{CC}$ \\
\hline Bromopsis erecta. subsp. microchaeta (Font & End Alg-Mar & Hemi & $\mathrm{C}$ \\
\hline
\end{tabular}


Bulletin de la Société Royale des Sciences de Liège, Vol. : 91, articles, 2021, p. 317 - 360

\begin{tabular}{|c|c|c|c|}
\hline Quer). H. Scholz \& Valdès. & & & \\
\hline Cynosurus balansae Coss \& Dur. & End Alg-Mar & Hemi & $\mathrm{AC}$ \\
\hline Cynosurus effusus Link & Sténomed & Ther & $\mathrm{C}$ \\
\hline Dactylis glomerata L., sensu lato & Paleotemp & Hemi & $\mathrm{C}$ \\
\hline Echinaria capitata (L.) Desf. & Atlantique-Med & Ther & $\mathrm{C}$ \\
\hline Elymus panormitanus (Parl) Tzvelev & E. Med & Hemi & $\mathrm{R}$ \\
\hline Festuca circummediterranea Patzke & Circumboreal & Hemi & $\mathrm{R}$ \\
\hline $\begin{array}{l}\text { Helictochlotoa cincinnata (Ten.) Romero } \\
\text { Zarco. }\end{array}$ & Stenomed & Hemi & $\mathrm{R}$ \\
\hline Holcus lanatus L. & Boreal & Hemi & $\mathrm{C}$ \\
\hline Hordeum bulbosum L. & Sub-tropical & Hemi & $\mathrm{AC}$ \\
\hline Lagurus ovatus L. & Eurymed & Ther & $\mathrm{CC}$ \\
\hline Lolium perenne L. & Boreal & Hemi & $\mathrm{C}$ \\
\hline Melica cupanii Guss. & Méd-Ir-Tour & Hemi & $\mathrm{AC}$ \\
\hline Melica uniflora Retz. & Paleotemp & Hemi & AR \\
\hline Melica ciliata $\mathrm{L}$. & Euras & Hemi & $\mathrm{C}$ \\
\hline Patzkea patula (Desf.) H. Scholz. & Stenomed & Hemi & $\mathrm{AC}$ \\
\hline Patzkea durandoi (Clausson) G. H. Loos. & End N.Afr. & Hemi & $\mathrm{AR}$ \\
\hline Phleum phleoides (L.) H. Karst. & Europ-Sib & Hemi & AR \\
\hline Poa bulbosa L. subsp. bulbosa & Paleotemp & Hemi & $\mathrm{C}$ \\
\hline $\begin{array}{l}\text { Trisetum flavescens (L.) P. Beauv. subsp. } \\
\text { flavescens }\end{array}$ & Euras & Hemi & $\mathrm{AC}$ \\
\hline Vulpia sicula (Presl.) Link. & Méd-Mont & Hemi & $\mathrm{C}$ \\
\hline \multicolumn{4}{|l|}{ Apiaceae } \\
\hline Ammoides atlantica Coss. \& Dur. & End Alg & Hemi & $\mathrm{AC}$ \\
\hline Anthriscus silvestris Hoffm. & Paleotemp & Hemi & $\mathrm{R}$ \\
\hline Binium alpinum Waldest \& Kit. & Med & Geo & $\mathrm{CC}$ \\
\hline Bupleurum montanum Coss. & End Alg-Mar & Hemi & $\mathrm{AR}$ \\
\hline
\end{tabular}


Bulletin de la Société Royale des Sciences de Liège, Vol. : 91, articles, 2021, p. 317 - 360

\begin{tabular}{|c|c|c|c|}
\hline Bupleurum spinosum Gouan. & Eurymed & Cham & $\mathrm{R}$ \\
\hline Chaerophyllum temulum $\mathrm{L}$. & Euras & Hemi & $\mathrm{R}$ \\
\hline Conopodium glaberimum (Desf.) Engstrand. & End N.Afr. & Geo & $\mathrm{C}$ \\
\hline Eryngium tricuspidatum subsp. tricuspidatum & S.W Méd & Hemi & $\mathrm{CC}$ \\
\hline Ferula communis L. subsp. communis & Stenomed & Hemi & $\mathrm{CC}$ \\
\hline $\begin{array}{l}\text { Heracleum sphondylium L. subsp. algeriense } \\
\text { (Batt. \& Trab.) Dobignard }\end{array}$ & End Alg & Hemi & $\mathrm{R}$ \\
\hline Pimpinella battandieri Chabert. & End Alg & Hemi & $\mathrm{R}$ \\
\hline $\begin{array}{l}\text { Physospermum verticillatum (Waldst. \& Kit.) } \\
\text { Vis. }\end{array}$ & Med-Mont & Hemi & $\mathrm{R}$ \\
\hline Sanicula europea L. & Euras & Hemi & $\mathrm{AC}$ \\
\hline Scandix pecten-veneris L. & Eurymed & Ther & $\mathrm{CC}$ \\
\hline Selinopsis montana Coss. \& Durieu ex Batt. & End Alg-Tun & Geo & AR \\
\hline Smyrnium olusatrum L. & Atlantique-Med & Hemi & $\mathrm{CC}$ \\
\hline $\begin{array}{l}\text { Torilis arvensis (Huds.) Link subsp. neglecta } \\
\text { (Spreng.) Thell. }\end{array}$ & Stenomed & Ther & $\mathrm{CC}$ \\
\hline Torilis nodosa (L.) Gaertn. subsp. nodosa & Euras & Ther & $\mathrm{CC}$ \\
\hline \multicolumn{4}{|l|}{ Rosaceae } \\
\hline Almanchier ovalus Medicus. & Stenomed & Phan & $\mathrm{R}$ \\
\hline Crataegus laciniata Ucria. & Med-Mont & Phan & AR \\
\hline Crataegus monogyna Jacq. & Paleotemp & Phan & $\mathrm{C}$ \\
\hline Potentilla micrantha Ramond. & Eurymed & Hemi & $\mathrm{AR}$ \\
\hline Potentilla caulescens L. & Oro-Med & Hemi & RR \\
\hline Prunus avium L. & Europ-Cauca & Phan & $\mathrm{AC}$ \\
\hline Prunus prostrata Labill & Med-Asiatique & Phan & $\mathrm{R}$ \\
\hline Rosa sempervirens $\mathrm{L}$. & Stenomed & Phan & $\mathrm{AC}$ \\
\hline Rosa canina L., sensu lato & Paleotemp & Phan & $\mathrm{C}$ \\
\hline Rosa micrantha Borrer ex Sm. & Eurymed & Phan & $\mathrm{AR}$ \\
\hline Rubus ulmifolius Scott. & Eurymed & Phan & $\mathrm{C}$ \\
\hline
\end{tabular}


Bulletin de la Société Royale des Sciences de Liège, Vol. : 91, articles, 2021, p. 317 - 360

\begin{tabular}{|c|c|c|c|}
\hline Rubus incanescens (DC.) Bertol. & W. Europe & Phan & $\mathrm{AC}$ \\
\hline $\begin{array}{l}\text { Sanguisorba minor subsp. baléarica (Nyman) } \\
\text { Munoz Garm. \& C. Navarro. }\end{array}$ & Euras & Hemi & $\mathrm{R}$ \\
\hline Sorbus aria (L.) Crantz & Paleotemp & Phan & $\mathrm{R}$ \\
\hline Sorbus torminalis (L.) Crantz & Euras & Phan & $\mathrm{R}$ \\
\hline \multicolumn{4}{|l|}{ Lamiaceae } \\
\hline Ajuga iva (L.) Schreber & Stenomed & Cham & $\mathrm{CC}$ \\
\hline Lamium flexuosum Ten. & Méd-Mont & Hemi & $\mathrm{AC}$ \\
\hline Melissa officinalis L. & Eurymed & Hemi & AR \\
\hline Mentha pulegium L. & Eurymed & Hemi & $\mathrm{AC}$ \\
\hline Mentha suaveolens Ehrh. & Atlantique-Med & Hemi & $\mathrm{CC}$ \\
\hline Micromeria graeca (L.) Benth \& Rchb. & Med & Cham & $\mathrm{CC}$ \\
\hline $\begin{array}{l}\text { Clinopodium vulgare L. subsp. arundanum } \\
\text { (Boiss.) Nyman }\end{array}$ & Stenomed & Hemi & $\mathrm{CC}$ \\
\hline $\begin{array}{l}\text { Origanum vulgare L. subsp. glandulosum } \\
\text { (Desf.) Letswaart }\end{array}$ & End Alg-Tun & Hemi & $\mathrm{C}$ \\
\hline Phlomis bovei De Noé & End Alg-Tun & Hemi & $\mathrm{R}$ \\
\hline Prunella vulgaris L. & Boreal & Hemi & $\mathrm{AR}$ \\
\hline Rosmarinus officinalis L. & Stenomed & Phan & $\mathrm{C}$ \\
\hline Salvia sclarea $\mathrm{L}$. & Eurymed & Cham & $\mathrm{R}$ \\
\hline Scuttelaria columnae All. & Méd-Mont & Hemi & $\mathrm{R}$ \\
\hline $\begin{array}{l}\text { Stachys officinalis subsp. algeriensis (de noé) } \\
\text { Franco }\end{array}$ & Europe & Hemi & $\mathrm{AC}$ \\
\hline Stachys circinnata L'Her & Med & Ther & $\mathrm{C}$ \\
\hline Thymus algériensis Boiss \& Reut. & End N.Afr. & Cham & $\mathrm{CC}$ \\
\hline Thymus dreatensis Batt. & End Alg & Cham & $\mathrm{RR}$ \\
\hline Teucrium atratum Pomel. & End Alg & Hemi & $\mathrm{R}$ \\
\hline Teucrium chamaedrys L. & Eurymed & Cham & $\mathrm{AC}$ \\
\hline Teucrium pseudo-scorodonia Desf. & W. Europe & Hemi & $\mathrm{AC}$ \\
\hline
\end{tabular}


Bulletin de la Société Royale des Sciences de Liège, Vol. : 91, articles, 2021, p. 317 - 360

\begin{tabular}{|c|c|c|c|}
\hline Brassicaceae & & & \\
\hline $\begin{array}{l}\text { Alliaria petiolata (M. Bieb.) Cavara \& } \\
\text { Grande. }\end{array}$ & Paleotemp & Hemi & $\mathrm{AC}$ \\
\hline Alyssum serpyllifolium Desf. & Oro-Med & Cham & AR \\
\hline Alyssum spinosum L. & Oro-W. Med & Cham & $\mathrm{RR}$ \\
\hline Arabidopsis thaliana (L.) Heynh. & Paleotemp & Ther & $\mathrm{CC}$ \\
\hline Arabis auriculata Lam & Oro-Méd & Ther & $\mathrm{AC}$ \\
\hline Arabis pubescens (Desf.) Poir. & End N.Afr. & Hemi & $\mathrm{AC}$ \\
\hline Arabis turrita $\mathrm{L}$. & S. Europe & Hemi & $\mathrm{R}$ \\
\hline $\begin{array}{l}\text { Arabis alpina L subsp. caucasica (Willd.) } \\
\text { Briq. }\end{array}$ & Méd-Mont & Hemi & AR \\
\hline Arabis hirsuta L. Scop. & Circumboreal & Ther & $\mathrm{R}$ \\
\hline Diplotaxis muralis (L.) DC & Europ & Ther & $\mathrm{R}$ \\
\hline Draba muralis L. & Boreal & Ther & $\mathrm{R}$ \\
\hline Draba hispanica & Med & Hemi & AR \\
\hline $\begin{array}{l}\text { Iberis atlantica (Litard. \& Maire) Greuter \& } \\
\text { Burdet }\end{array}$ & End Alg-Mar & Hemi & $\mathrm{R}$ \\
\hline Lobularia maritima (L). Desv. & Stenomed & Cham & $\mathrm{CC}$ \\
\hline Thlaspi perfoliatum L. subsp. perfoliatum & Paleotemp & Ther & $\mathrm{AC}$ \\
\hline \multicolumn{4}{|l|}{ Caryophyllaceae } \\
\hline Arenaria serpyllifolia L. subsp. serpyllifolia. & Cosmop & Ther & $\mathrm{C}$ \\
\hline $\begin{array}{l}\text { Cerastium brachypetalum. subsp. roeseri } \\
\text { (Boiss. \& Heldr) }\end{array}$ & Med-Tour & Ther & $\mathrm{R}$ \\
\hline Cerastium atlanticum Dur & End N.Afr. & Hemi & $\mathrm{R}$ \\
\hline Dianthus balbisii Ser. & Oro-Med & Hemi & $\mathrm{R}$ \\
\hline Dianthus serrulatus Desf. & Stenomed & Hemi & $\mathrm{AC}$ \\
\hline Herniaria hirsuta $\mathrm{L}$. & Paleotemp & Ther & $\mathrm{AC}$ \\
\hline Moehringia stellarioides Coss. & End Alg & Hemi & $\mathrm{R}$ \\
\hline $\begin{array}{l}\text { Paronychia arabica (L.) DC subsp. aurasiaca } \\
\text { (Webb.) Maire \& Weiller. }\end{array}$ & End N.Afr. & Hemi & $\mathrm{AC}$ \\
\hline
\end{tabular}


Bulletin de la Société Royale des Sciences de Liège, Vol. : 91, articles, 2021, p. 317 - 360

\begin{tabular}{|c|c|c|c|}
\hline Polycarpon bivonae (J. Gay) \& Maire. & W. Med & Hemi & $\mathrm{AC}$ \\
\hline Saponaria sicula Rafin. & C. Med & Hemi & $\mathrm{R}$ \\
\hline Silene secundiflora Ott. & Stenomed & Ther & $\mathrm{R}$ \\
\hline Silene pseudo-atocion Desf. & Stenomed & Ther & $\mathrm{C}$ \\
\hline Silene choulettii Coss. & End Alg & Hemi & $\mathrm{AC}$ \\
\hline Silene atlantica Coss. & End Alg-Tun & Hemi & $\mathrm{AC}$ \\
\hline Stellaria pallida (Dumort.) Piré. & Paleotempéré & Hemi & $\mathrm{C}$ \\
\hline Stellaria holostea L. & Europ-Caucas & Hemi & RR \\
\hline \multicolumn{4}{|l|}{ Scrophylariaceae } \\
\hline Kickxia lanigera (Desf.) Hand. - Mazz. & Med & Ther & AR \\
\hline $\begin{array}{l}\text { Linaria virgata Desf. subsp. algeriensis } \\
\text { Murb. }\end{array}$ & End Alg-Tun & Ther & $\mathrm{AC}$ \\
\hline Linaria reflexa Desf. & Eurymed & Ther & $\mathrm{CCC}$ \\
\hline Verbascum sinuatum L. & Eurymed & Ther & $\mathrm{CC}$ \\
\hline Veronica montana $\mathrm{L}$. & Europ & Hemi & $\mathrm{RR}$ \\
\hline Veronica praecox All. & C. Europ & Ther & $\mathrm{R}$ \\
\hline Veronica agrestis $\mathrm{L}$. & SW. Euras & Ther & $\mathrm{AC}$ \\
\hline \multicolumn{4}{|l|}{ Crassulaceae } \\
\hline Sedum sediforme (Jacq.) Pau. & Stenomed & Cham & $\mathrm{C}$ \\
\hline Sedum dasyphyllum L. subsp. dasyphyllum & Eurymed & Cham & $\mathrm{AC}$ \\
\hline Sedum pubescens Vahl. & End Alg-Tun & Ther & $\mathrm{AC}$ \\
\hline Sedum album L. & Euras & Cham & $\mathrm{C}$ \\
\hline Sedum multiceps Coss. \& Dur. & End Alg & Cham & $\mathrm{R}$ \\
\hline Sedum amplexicaule DC. & Oro-Med & Hemi & $\mathrm{R}$ \\
\hline Sedum acre L. & Euras & Cham & AR \\
\hline Sedum caeruleum L. & C. Med & Ther & $\mathrm{CC}$ \\
\hline Umbilicus rupestris (Salisb.) Dandy & Atlantique-Med & Geo & $\mathrm{AC}$ \\
\hline Liliaceae & & & \\
\hline
\end{tabular}


Bulletin de la Société Royale des Sciences de Liège, Vol. : 91, articles, 2021, p. 317 - 360

\begin{tabular}{|c|c|c|c|}
\hline $\begin{array}{l}\text { Allium sphaerocephalum L. subsp. } \\
\text { sphaerocephalum }\end{array}$ & Eurymed & Geo & $\mathrm{AC}$ \\
\hline Asphodelus ramosus L. subsp. ramosus & Stenomed & Geo & $\mathrm{CCC}$ \\
\hline Fritillaria oranensis Pomel & End N.Afr. & Geo & AR \\
\hline Muscari comosum (L.) Mill. & Med & Geo & $\mathrm{C}$ \\
\hline Oncostema elongata (Parl.) Speta. & W. Med & Geo & $\mathrm{C}$ \\
\hline Ornithogalum baeticum Boiss. & Atlantique-Med & Geo & $\mathrm{C}$ \\
\hline Ruscus aculeatus L. & Atlantique-Med & Cham & $\mathrm{C}$ \\
\hline Smilax aspera $\mathrm{L}$. & Sub-tropical & Phan & $\mathrm{AC}$ \\
\hline Tulipa sylvestris L. subsp. australis Link. & Eurymed & Geo & $\mathrm{CC}$ \\
\hline \multicolumn{4}{|l|}{ Ranunculaceae } \\
\hline Clematis cirrhosa $\mathrm{L}$. & Stenomed & Phan & $\mathrm{C}$ \\
\hline Delphinium perigrinum L. & Stenomed & Hemi & $\mathrm{AC}$ \\
\hline Nigella damascenna $\mathrm{L}$. & Eurymed & Ther & $\mathrm{AC}$ \\
\hline Ranunculus bulbosus L. & Euras & Hemi & $\mathrm{R}$ \\
\hline $\begin{array}{l}\text { Ranunculus spicatus. subsp. maroccanus } \\
\text { (Coss.) Greuter \& Burdet. }\end{array}$ & End Alg-Mar & Geo & $\mathrm{C}$ \\
\hline Ranunculus ficariiformis (F. W.) Schultz. & Euras & Hemi & $\mathrm{AC}$ \\
\hline \multicolumn{4}{|l|}{ Polypodiaceae } \\
\hline Athyrium felix-femina (L.) Roth & Circumbor & Hemi & $\mathrm{AR}$ \\
\hline Pteridium aquilinum (L.) Kuhn. & Cosmopolite & Geo & $\mathrm{CC}$ \\
\hline Polypodium cambricum. Willd. & Atlantique-Med & Hemi & $\mathrm{CC}$ \\
\hline Polystichum setiferum (Forskal) Woynar & Macar-Euras & Geo & $\mathrm{R}$ \\
\hline \multicolumn{4}{|l|}{ Orchidaceae } \\
\hline $\begin{array}{l}\text { Anacamptis coriophora subsp. fragrans } \\
\text { (Pollini). R.M. Bateman, Pridgeon \& M.W. } \\
\text { Chase }\end{array}$ & Med & Geo & $\mathrm{AC}$ \\
\hline $\begin{array}{l}\text { Anacamptis morio subsp. longicornu (Poir.) } \\
\text { H. Kretzschmar, Eccarius \& H.Dietr. }\end{array}$ & Euras & Geo & $\mathrm{R}$ \\
\hline Anacamptis papilionacea (L.) R.M. Bateman, & Med & Geo & AR \\
\hline
\end{tabular}


Bulletin de la Société Royale des Sciences de Liège, Vol. : 91, articles, 2021, p. 317 - 360

\begin{tabular}{|c|c|c|c|}
\hline Pridgeon \& M.W. Chase & & & \\
\hline $\begin{array}{l}\text { Androrchis olbiensis (Reut. Ex Gren.) D. } \\
\text { Tyteca \& E. Klein. }\end{array}$ & Euras & Geo & AR \\
\hline $\begin{array}{l}\text { Androrchis patens (Desf.) D. Tyteca \& E. } \\
\text { Klein. }\end{array}$ & End Alg-Tun & Geo & AR \\
\hline $\begin{array}{l}\text { Androrchis pauciflora subsp. laeta (Steinh.) } \\
\text { Véla, Rebbas \& R. Martin }\end{array}$ & End Alg-Tun & Geo & $\mathrm{R}$ \\
\hline Cephalanthera longifolia (L.) Fritsch. & Euras & Geo & $\mathrm{AC}$ \\
\hline $\begin{array}{l}\text { Dactylorhiza munbyana (Boiss. \& Reut.) } \\
\text { Aver. }\end{array}$ & End N.Afr. & Geo & $\mathrm{AC}$ \\
\hline $\begin{array}{l}\text { Himantoglossum robertianum (Loisel.) P. } \\
\text { Delforge }\end{array}$ & Stenomed & Geo & $\mathrm{AC}$ \\
\hline Himantoglossum hircinum (L.) Spreng. & Eurymed & Geo & AR \\
\hline Limodorum arbortivum (L). Sw. & Euras & Geo & $\mathrm{AC}$ \\
\hline $\begin{array}{l}\text { Neotinea tridentata subsp. conica (Willd.) } \\
\text { R.M. Bateman, Pridgeon \& M. W. Chase }\end{array}$ & Euras & Geo & $\mathrm{AC}$ \\
\hline Ophrys battandieri G. Camus & End N.Afr. & Geo & $\mathrm{R}$ \\
\hline Ophrys fusca Link. subsp. fusca & Eurymed & Geo & $\mathrm{C}$ \\
\hline Ophrys lutea (Cav.) Gouan. & Med & Geo & $\mathrm{C}$ \\
\hline Ophrys numida Devillers-Tersch. \& Devillers & End Alg-Tun & Geo & $\mathrm{R}$ \\
\hline Ophrys speculum Link & Stenomed & Geo & $\mathrm{AC}$ \\
\hline $\begin{array}{l}\text { Ophrys tenthredinifera subsp. tenthredinifera } \\
\text { Willd. }\end{array}$ & Stenomed & Geo & $\mathrm{C}$ \\
\hline Orchis anthropophora (L.) All. & Atlantique-Med & Geo & $\mathrm{C}$ \\
\hline Orchis italica Poir. & Euras & Geo & $\mathrm{C}$ \\
\hline Orchis purpurea Huds. subsp. purpurea & Euras & Geo & $\mathrm{R}$ \\
\hline \multicolumn{4}{|l|}{ Plantaginaceae } \\
\hline Plantago serraria $\mathrm{L}$. & Stenomed & Hemi & $\mathrm{CC}$ \\
\hline Plantago bellardii L. & Stenomed & Ther & $\mathrm{CC}$ \\
\hline Plantago coronopus L. & Euras & Hemi & $\mathrm{AC}$ \\
\hline
\end{tabular}


Bulletin de la Société Royale des Sciences de Liège, Vol. : 91, articles, 2021, p. 317 - 360

\begin{tabular}{|c|c|c|c|}
\hline Plantago major L. subsp. major & Euras & Hemi & $\mathrm{CC}$ \\
\hline \multicolumn{4}{|l|}{ Geraniaceae } \\
\hline Erodium battandieranum Rouy. & End Alg & Hemi & $\mathrm{R}$ \\
\hline Erodium aethiopicum $\mathrm{L}$. & Med & Ther & $\mathrm{C}$ \\
\hline Geranium purpurum Vill. & Cosmp & Ther & $\mathrm{CC}$ \\
\hline Geranium atlanticum Boiss. \& Reut. & End N.Afr. & Hemi & $\mathrm{C}$ \\
\hline Geranium lucidum L. & Atlantique-Med & Ther & $\mathrm{C}$ \\
\hline Geranium molle L. & Euras & Ther & $\mathrm{CC}$ \\
\hline \multicolumn{4}{|l|}{ Rubiaceae } \\
\hline Asperula laevigata L. & Stenomed & Hemi & $\mathrm{CC}$ \\
\hline Asperula hirsuta Desf. & W. Med & Hemi & $\mathrm{CC}$ \\
\hline Gallium tunetanum Lam. & S. W. Med & Hemi & $\mathrm{CC}$ \\
\hline Galium spurium (L.) Hartm. & Euras & Ther & $\mathrm{CC}$ \\
\hline Gallium mollugo subsp. erectum Syme & Euras & Hemi & $\mathrm{R}$ \\
\hline Sherardia arvensis $\mathrm{L}$. & Euras & Ther & $\mathrm{CC}$ \\
\hline \multicolumn{4}{|l|}{ Campanulaceae } \\
\hline Campanula trichocalycina (Ten). & Med-Mont & Hemi & $\mathrm{R}$ \\
\hline Campanula rapunculus L. & Paleotemp & Hemi & $\mathrm{C}$ \\
\hline $\begin{array}{l}\text { Campanula trachelium L. subsp. mauritanica } \\
\text { (Pomel) Quezel. }\end{array}$ & End Alg-Mar & Hemi & $\mathrm{AC}$ \\
\hline Campanula dichotoma $\mathrm{L}$. & Med & Ther & $\mathrm{C}$ \\
\hline \multicolumn{4}{|l|}{ Dipsacaceae } \\
\hline Knautia mauritanica Pomel. & End N.Afr. & Hemi & $\mathrm{AC}$ \\
\hline Scabiosa columbaria L. & Euras & Hemi & $\mathrm{R}$ \\
\hline Scabiosa semipapposa Salzm. & Eurymed & Ther & $\mathrm{CC}$ \\
\hline Scabiosa stellata L. & W.Med & Ther & $\mathrm{CC}$ \\
\hline \multicolumn{4}{|l|}{ Euphorbiaceae } \\
\hline Euphorbia amygdaloides L. & Euras & Cham & $\mathrm{R}$ \\
\hline
\end{tabular}


Bulletin de la Société Royale des Sciences de Liège, Vol. : 91, articles, 2021, p. 317 - 360

\begin{tabular}{|c|c|c|c|}
\hline Euphorbia pterococca Brot & Stenomed & Ther & $\mathrm{AC}$ \\
\hline Euphorbia peplus L. & Cosmopolite & Ther & $\mathrm{CC}$ \\
\hline \multicolumn{4}{|l|}{ Primulaceae } \\
\hline Anagallis monelli L. subsp. monelli & Stenomed & Hemi & $\mathrm{C}$ \\
\hline Cyclamen africanum Boiss. \& Reut. & End Alg-Tun & Geo & $\mathrm{C}$ \\
\hline $\begin{array}{l}\text { Lysimachia arvensis (L.) U. Manns \& } \\
\text { Anderb. }\end{array}$ & Cosmopolite & Ther & $\mathrm{CC}$ \\
\hline Lysimachia cousiniana Coss \& Dur. & End Alg-Tun & Hemi & $\mathrm{AC}$ \\
\hline $\begin{array}{l}\text { Primula acaulis L. subsp. atlantica (Maire \& } \\
\text { Wilczek) Greuter \& Burdet. }\end{array}$ & End Alg-Mar & Hemi & $\mathrm{R}$ \\
\hline \multicolumn{4}{|l|}{ Violaceae } \\
\hline Viola odorata L. & Eurymed & Hemi & $\mathrm{R}$ \\
\hline Viola mumbyana Boiss. \& Reut. & End N.Afr. & Hemi & $\mathrm{AC}$ \\
\hline Viola riviniana $\mathrm{Rchb}$. & Europ & Hemi & $\mathrm{R}$ \\
\hline \multicolumn{4}{|l|}{ Convolvulaceae } \\
\hline Convolvulus elegantissimus Mill. & Stenomed & Hemi & $\mathrm{CC}$ \\
\hline Convolvulus cantabrica $\mathrm{L}$. & Eurymed & Hemi & $\mathrm{AC}$ \\
\hline Convolvulus lineatus L. & Circumboréa & Hemi & $\mathrm{AC}$ \\
\hline \multicolumn{4}{|l|}{ Hypericaceae } \\
\hline Hypericum montanum L. & Europ-Caucas & Hemi & $\mathrm{R}$ \\
\hline Hypericum perforatum $\mathrm{L}$. & Paleotemp & Hemi & $\mathrm{C}$ \\
\hline Hypericum tomentosum $\mathrm{L}$. & W. Med & Hemi & $\mathrm{CC}$ \\
\hline \multicolumn{4}{|l|}{ Polygonaceae } \\
\hline $\begin{array}{l}\text { Rumex acetosella L. subsp. angiocarpus } \\
\text { Murb. }\end{array}$ & Sub-Cosmopolite & Hemi & $\mathrm{AC}$ \\
\hline Rumex tuberosus L. & Med-Tour & Hemi & $\mathrm{C}$ \\
\hline $\begin{array}{l}\text { Rumex bucephalophorus L. subsp. gallicus } \\
\text { (Steinh) Rech. }\end{array}$ & Stenomed & Ther & $\mathrm{AC}$ \\
\hline Caprifoliaceae & & & \\
\hline
\end{tabular}


Bulletin de la Société Royale des Sciences de Liège, Vol. : 91, articles, 2021, p. 317 - 360

\begin{tabular}{|c|c|c|c|}
\hline Lonicera kabylica Rehder & End Alg & Phan & $\mathrm{R}$ \\
\hline Lonicera etrusca Santi. & Eurymed & Phan & $\mathrm{R}$ \\
\hline Lonicera implexa Aiton. & Eurymed & Phan & $\mathrm{C}$ \\
\hline Sambucus ebulus L. & Eurymed & Hemi & $\mathrm{R}$ \\
\hline Sambucus nigra L. & S. Europ & Hemi & $\mathrm{R}$ \\
\hline Viburnum lantana $\mathrm{L}$. & Oro-Med & Phan & RR \\
\hline \multicolumn{4}{|l|}{ Cyperaceae } \\
\hline Carex dystachia Desf. & Stenomed & Hemi & $\mathrm{C}$ \\
\hline Carex olbiensis Jord. & Euras & Hemi & $\mathrm{R}$ \\
\hline $\begin{array}{l}\text { Carex sylvatica subsp. paui (sennen) A. Bolos } \\
\& \text { O. Bolos }\end{array}$ & Europ & Hemi & $\mathrm{R}$ \\
\hline \multicolumn{4}{|l|}{ Urticaceae } \\
\hline Urtica dioica $\mathrm{L}$. & Cosmop & Hemi & $\mathrm{AC}$ \\
\hline Urtica membranacea Poir. & Med & Ther & $\mathrm{CC}$ \\
\hline \multicolumn{4}{|l|}{ Apocynaceae } \\
\hline Nerium oleander L. & Stenomed & Phan & $\mathrm{C}$ \\
\hline Vincetoxicum Hirundinaria Medik. & Euras & Hemi & $\mathrm{C}$ \\
\hline \multicolumn{4}{|l|}{ Valerianaceae } \\
\hline Centranthus calcitrapa (L.) Dufr. & Stenomed & Ther & $\mathrm{CC}$ \\
\hline Centranthus ruber DC & Eurymed & Hemi & $\mathrm{CC}$ \\
\hline $\begin{array}{l}\text { Fedia gracilliflora Fisch. \& C.A. Mey, sensu } \\
\text { lato }\end{array}$ & Stenomed & Ther & $\mathrm{CC}$ \\
\hline Valeriana tuberosus L. & Med-Mont & Hemi & $\mathrm{AC}$ \\
\hline $\begin{array}{l}\text { Valerianella locustra F. carinata (Loisel.) } \\
\text { Devesa }\end{array}$ & Euras & Ther & $\mathrm{CC}$ \\
\hline \multicolumn{4}{|l|}{ Thymelaceae } \\
\hline Daphne gnidium L. & Stenomed & Phan & $\mathrm{C}$ \\
\hline Daphne laureola L. & Euras & Phan & $\mathrm{R}$ \\
\hline Salicaceae & & & \\
\hline
\end{tabular}


Bulletin de la Société Royale des Sciences de Liège, Vol. : 91, articles, 2021, p. 317 - 360

\begin{tabular}{|c|c|c|c|}
\hline Populus alba $\mathrm{L}$. & Paleotemp & Phan & $\mathrm{AC}$ \\
\hline Populus nigra $\mathrm{L}$. & Paleotemp & Phan & AR \\
\hline \multicolumn{4}{|l|}{ Aceraceae } \\
\hline $\begin{array}{l}\text { Acer monspessulanum L. subsp.martini (Jord) } \\
\text { P. Four. }\end{array}$ & Med & Phan & $\mathrm{RR}$ \\
\hline Acer obtusatum Waldst. \& Kit. Ex Willd. & S. Europe & Phan & $\mathrm{R}$ \\
\hline \multicolumn{4}{|l|}{ Araceae } \\
\hline Arum italicum Mill. & Stenomed & Geo & $\mathrm{C}$ \\
\hline Arisarum vulgare O. Targ. Tozz, sensu lato & Stenomed & Geo & $\mathrm{C}$ \\
\hline \multicolumn{4}{|l|}{ Gentianaceae } \\
\hline $\begin{array}{l}\text { Blackstonia perfoliata L. subsp.grandiflora } \\
\text { (Viv.) Maire }\end{array}$ & Med & Ther & $\mathrm{CC}$ \\
\hline $\begin{array}{l}\text { Centaurium erythraea subsp. suffruticosum } \\
\text { (Salzm. Ex Griseb.) }\end{array}$ & Paleotemp & Ther & $\mathrm{CC}$ \\
\hline \multicolumn{4}{|l|}{ Fagaceae } \\
\hline Quercus ilex subsp. ballota (Desf.) Samp. & Med & Phan & $\mathrm{C}$ \\
\hline Quercus canariensis Willd. & Atlantique-Med & Phan & $\mathrm{AC}$ \\
\hline Quercus suber L. & W. Med & Phan & $\mathrm{C}$ \\
\hline \multicolumn{4}{|l|}{ Papaveraceae } \\
\hline Chelidonium majus L. & Euras & Hemi & $\mathrm{R}$ \\
\hline Papaver rhoeas L. & E. Med & Ther & $\mathrm{CC}$ \\
\hline \multicolumn{4}{|l|}{ Iridaceae } \\
\hline Gladiolus dubius Guss. X italicus Mill. & Stenomed & Geo & $\mathrm{C}$ \\
\hline Iris inguicularis Poir. subsp. unguicularis & End Alg-Tun & Geo & $\mathrm{CC}$ \\
\hline Romulea lingustica (Parl.) & Med & Geo & $\mathrm{C}$ \\
\hline \multicolumn{4}{|l|}{ Linaceae } \\
\hline Linum gallicum L. & Stenomed & Ther & $\mathrm{C}$ \\
\hline Linum strictum L. & Stenomed & Ther & $\mathrm{AC}$ \\
\hline Boraginaceae & & & \\
\hline
\end{tabular}


Bulletin de la Société Royale des Sciences de Liège, Vol. : 91, articles, 2021, p. 317 - 360

\begin{tabular}{|c|c|c|c|}
\hline Cerinthe major L. & Stenomed & Hemi & $\mathrm{CC}$ \\
\hline Cynoglossum dioscoridis Vill. & W. Europe & Ther & $\mathrm{C}$ \\
\hline Cynoglossum cheirofolium $\mathrm{L}$. & Eurymed & Ther & $\mathrm{C}$ \\
\hline Myosotis speciosa Pomel & Paleotemp & Hemi & $\mathrm{AC}$ \\
\hline $\begin{array}{l}\text { Onosma tricerosperma subsp.mauritanica } \\
\text { (Maire) G. lopez }\end{array}$ & SW Europ & Hemi & AR \\
\hline \multicolumn{4}{|l|}{ Cistaceae } \\
\hline Cistus salvifolius L. & Stenomed & Phan & $\mathrm{CC}$ \\
\hline Fumana thymifolia (L.) Webb. & Stenomed & Cham & $\mathrm{AC}$ \\
\hline Helianthemum helianthemoides Desf. & End N.Afr. & Cham & RR \\
\hline Helianthemum cinereum (Cav.) Pers. & Eurymed & Cham & $\mathrm{C}$ \\
\hline \multicolumn{4}{|l|}{ Juncaceae } \\
\hline Luzula forsteri (Sm.) DC. & Eurymed & Geo & $\mathrm{AC}$ \\
\hline \multicolumn{4}{|l|}{ Malvaceae } \\
\hline Malva sylvestris L. & Cosmopolite & Hemi & $\mathrm{CC}$ \\
\hline Malope malachoides L. & Stenomed & Hemi & $\mathrm{C}$ \\
\hline \multicolumn{4}{|l|}{ Onagraceae } \\
\hline Circaea lutetiana $\mathrm{L}$. & Boreal & Hemi & $\mathrm{AC}$ \\
\hline Epilobium lanceolatum Seb. \& Maur. & Euras & Hemi & $\mathrm{R}$ \\
\hline \multicolumn{4}{|l|}{ Araliaceae } \\
\hline Hedera algeriensis Hibberd. & End Alg-Tun & Phan & $\mathrm{C}$ \\
\hline \multicolumn{4}{|l|}{ Fumariaceae } \\
\hline Fumaria capreolata L. subsp.capreolata & Eurymed & Ther & $\mathrm{CC}$ \\
\hline \multicolumn{4}{|l|}{ Paeoniaceae } \\
\hline $\begin{array}{l}\text { Paeonia mascula (L.) Mill. subsp. atlantica } \\
\text { (Cosson.) Greuter \& Burdet. }\end{array}$ & End Alg & Geo & $\mathrm{C}$ \\
\hline \multicolumn{4}{|l|}{ Berberidaceae } \\
\hline Epimedium perralderanium Coss. & End Alg & Hemi & RR \\
\hline
\end{tabular}


Bulletin de la Société Royale des Sciences de Liège, Vol. : 91, articles, 2021, p. 317 - 360

\begin{tabular}{|c|c|c|c|}
\hline Plumbaginaceae & & & \\
\hline Armeria alliacea (Cav.) Hoffm & S.W. Europe & Hemi & $\mathrm{R}$ \\
\hline \multicolumn{4}{|l|}{ Saxifragaceae } \\
\hline Saxifraga carpetana Boiss. \& Reut. & Eurymed & Hemi & AR \\
\hline \multicolumn{4}{|l|}{ Rhamnaceae } \\
\hline Rhamnus myrtifolius Willk. & Stenomed & Phan & $\mathrm{R}$ \\
\hline Rhamnus alpina L. & Oro-w. Med & Phan & $\mathrm{AR}$ \\
\hline \multicolumn{4}{|l|}{ Amaryllidaceae } \\
\hline Narcissus tazetta L., sensu lato & Stenomed & Geo & $\mathrm{CC}$ \\
\hline \multicolumn{4}{|l|}{ Taxaceae } \\
\hline Taxus baccata $\mathrm{L}$. & Paleo-temp & Phan & $\mathrm{AR}$ \\
\hline \multicolumn{4}{|l|}{ Cupressaceae } \\
\hline Juniperus oxycedrus L., sensu lato & Atlantique-Med & Phan & $\mathrm{CC}$ \\
\hline $\begin{array}{l}\text { Juniperus phoenicea L. subsp. turbinata } \\
\text { (Guss.) }\end{array}$ & Stenomed & Phan & $\mathrm{AC}$ \\
\hline \multicolumn{4}{|l|}{ Pinaceae } \\
\hline Cedrus atlantica (Endl.) Carrière & End Alg-Mar & Phan & $\mathrm{AC}$ \\
\hline \multicolumn{4}{|l|}{ Resedaceae } \\
\hline Reseda luteola $\mathrm{L}$. & Euras & Ther & $\mathrm{AC}$ \\
\hline Reseda alba $\mathrm{L}$. & Euras & Hemi & $\mathrm{C}$ \\
\hline \multicolumn{4}{|l|}{ Coriariaceae } \\
\hline Coriaria myrtifolia L. & W. Med & Phan & $\mathrm{AR}$ \\
\hline \multicolumn{4}{|l|}{ Ericaceae } \\
\hline Erica scoparia L. & Stenomed & Phan & AR \\
\hline \multicolumn{4}{|l|}{ Aquifoliaceae } \\
\hline Ilex aquifolium L. & Europ & Phan & $\mathrm{AC}$ \\
\hline \multicolumn{4}{|l|}{ Anacardiaceae } \\
\hline Pistacia terebinthus L. & Eurymed & Phan & $\mathrm{AC}$ \\
\hline
\end{tabular}


Bulletin de la Société Royale des Sciences de Liège, Vol. : 91, articles, 2021, p. 317 - 360

\begin{tabular}{|l|l|l|l|}
\hline Dioscoreaceae & & & \\
\hline Dioscorea communis (L.) Caddick \& Wilkin & Eurymed & Geo & AC \\
\hline Equisetaceae & & & \\
\hline Equisetum telmateia Ehrh. & Boreal & Geo & AC \\
\hline Oleaceae & & & \\
\hline Fraxinus angustifolia Vahl. & S. Europe & Phan & C \\
\hline Orobanchaceae & & & \\
\hline Orobanche schultzii Mutel & W. Europ & Geo & AC \\
\hline Orobanche rapum genistae & W. Europ & Geo & RR \\
\hline Orobanche ramosa L. & Tropical & Geo & AR \\
\hline Aspleniaceae & & Heomi & AR \\
\hline Asplenium trichomanes L. & Cosmopolite & Hemi & C \\
\hline Asplenium obovatum Viv. & Stenomed & Hemi & Hemi \\
\hline Asplenium cetarach L. & Euras & Hemi & \\
\hline Asplenium onopteris L. & & & \\
\hline
\end{tabular}


Bulletin de la Société Royale des Sciences de Liège, Vol. : 91, articles, 2021, p. 317 - 360

Annexe 2: Photographies de certaines espèces rares et /ou endémiques des massifs étudiés.

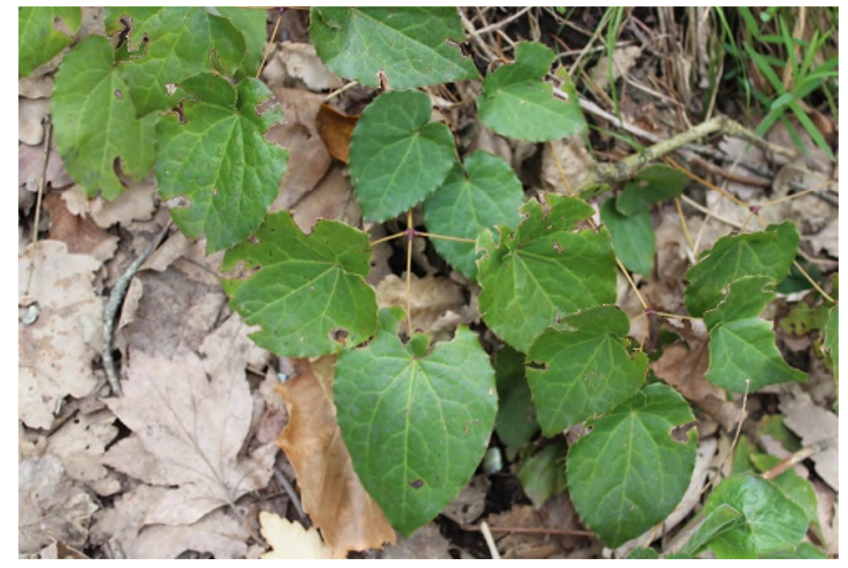

Figure 1 : Epimedium perralderanium Coss.

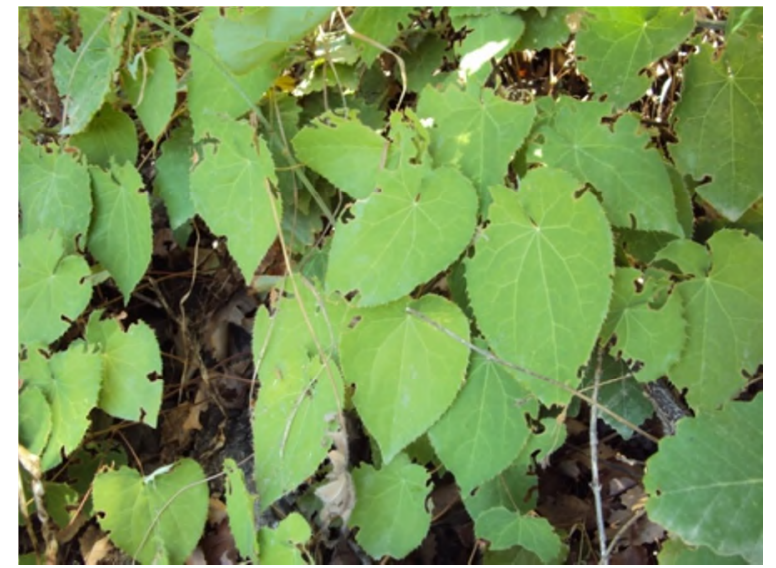

Figure 2 : Epimedium perralderanium Coss.

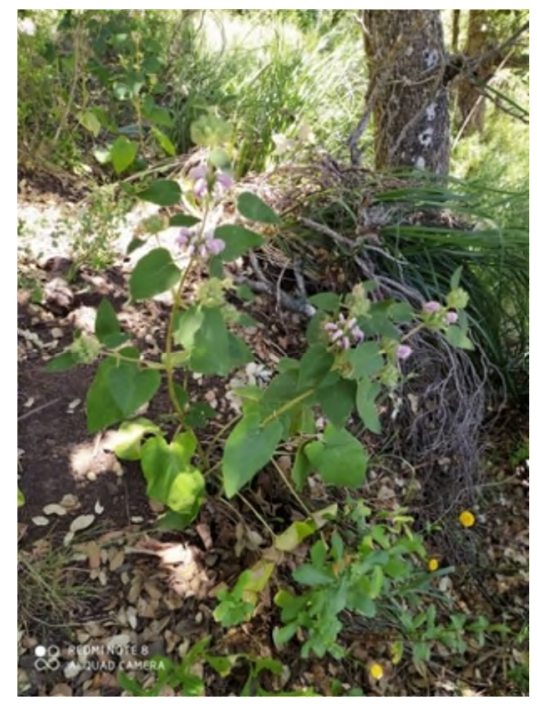

Figure 3 : Phlomis bovei De Noé

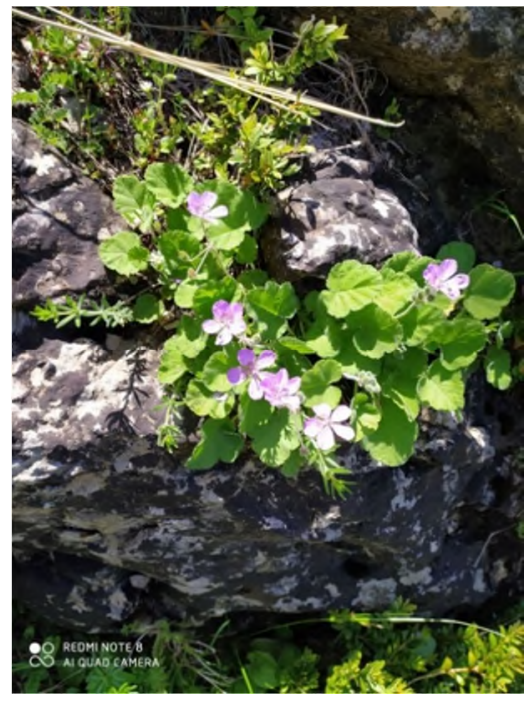

Figure 4 : Erodium battandieranum Rouy

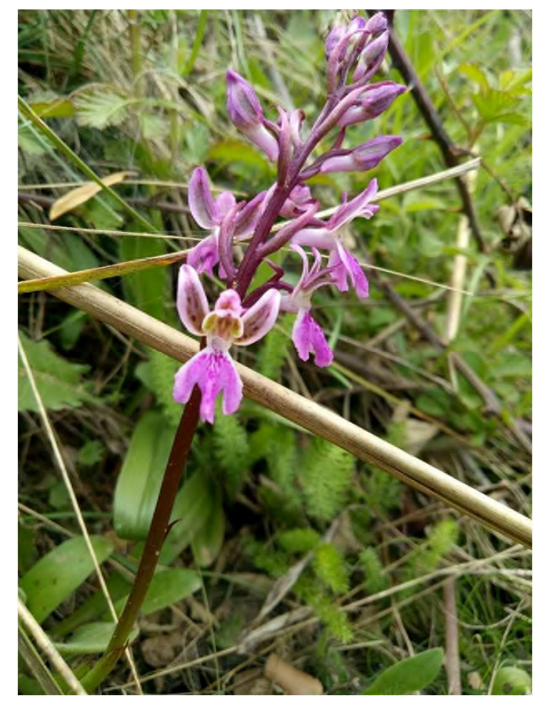

Figure 5 : Androrchis patens Desf. 


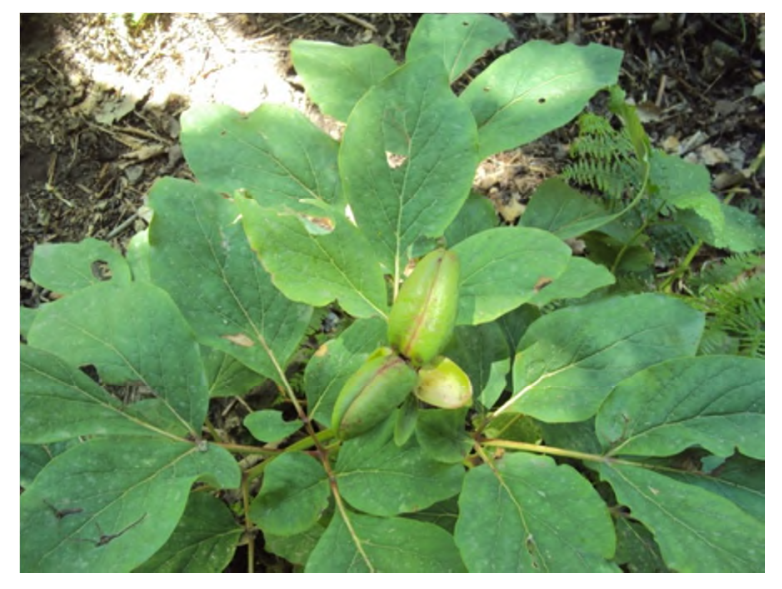

Figure 6 : Paeonia mascula (L.) Mill. subsp. atlantica (Cosson.) Greuter \& Burdet

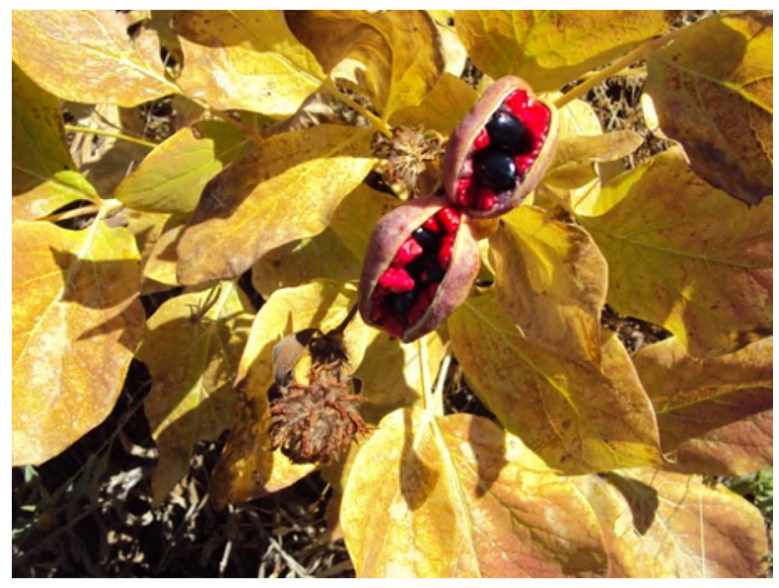

Figure 7 : Paeonia mascula (L.) Mill. subsp. atlantica (Cosson.) Greuter \& Burdet

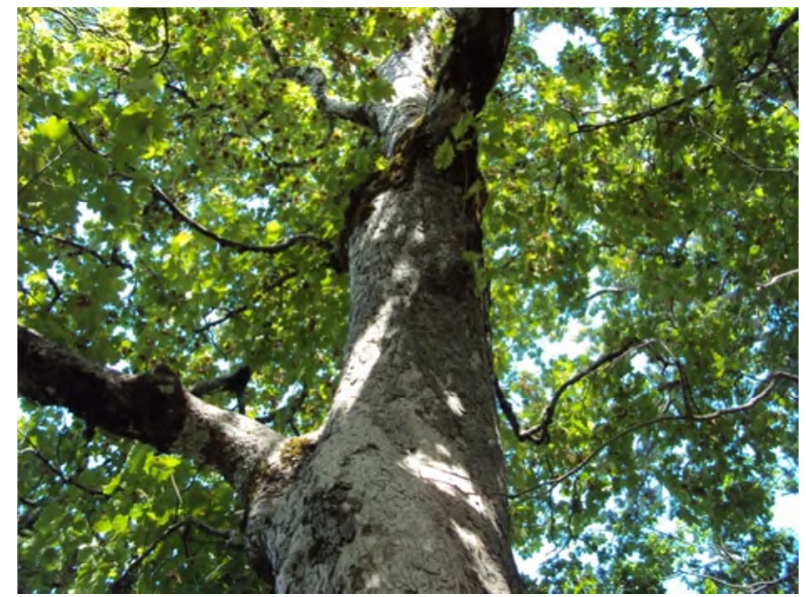

Figure 8 : Acer obtusatum Waldst. \& Kit. Ex Willd.

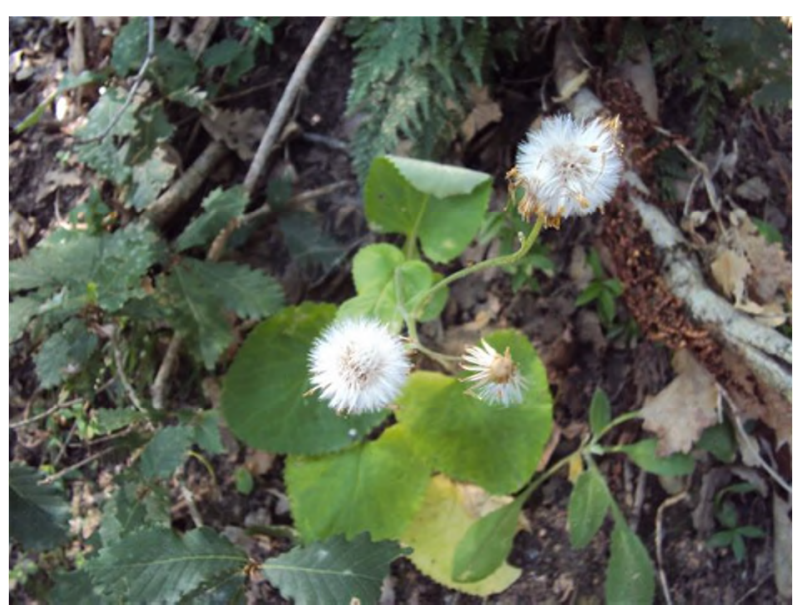

Figure 9 : Senecio perralderianus Coss. \& Dur. 
Bulletin de la Société Royale des Sciences de Liège, Vol. : 91, articles, 2021, p. 317 - 360

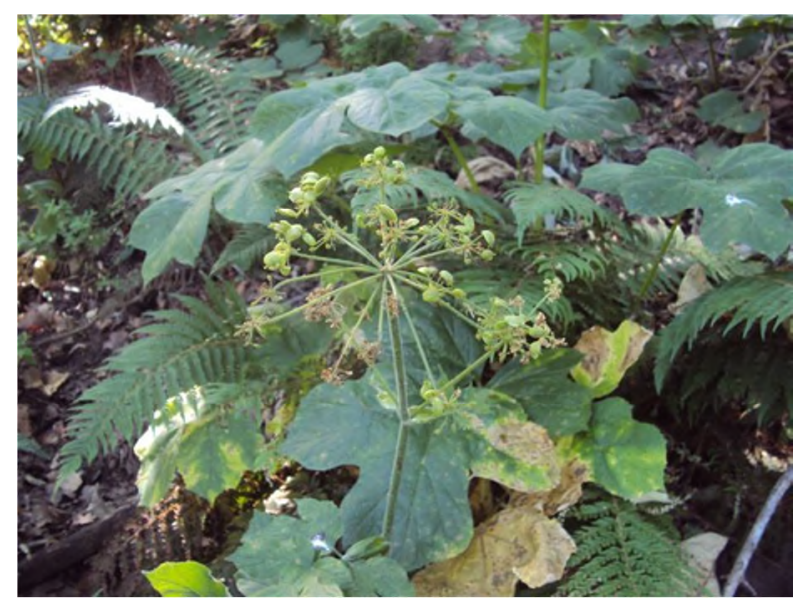

Figure 10 : Heracleum sphondylium L. subsp. algeriense

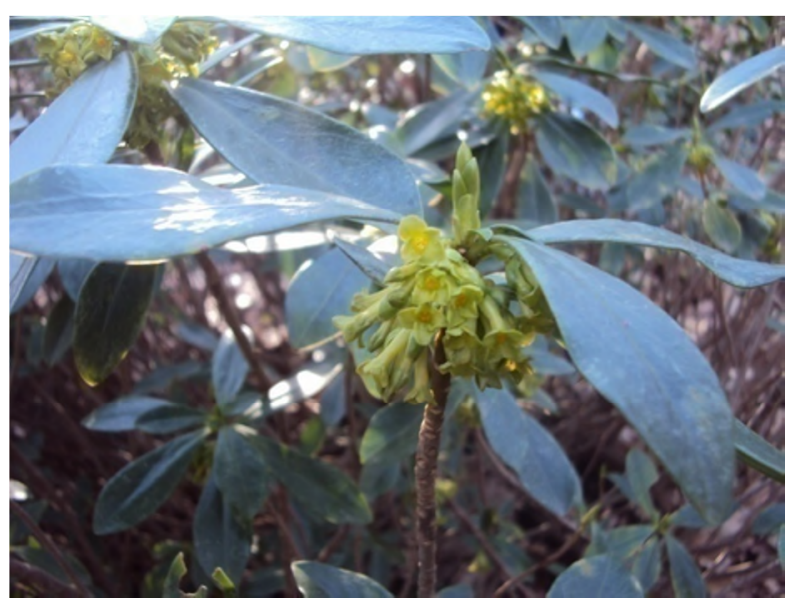

Figure 11 : Daphne laureola Coss.

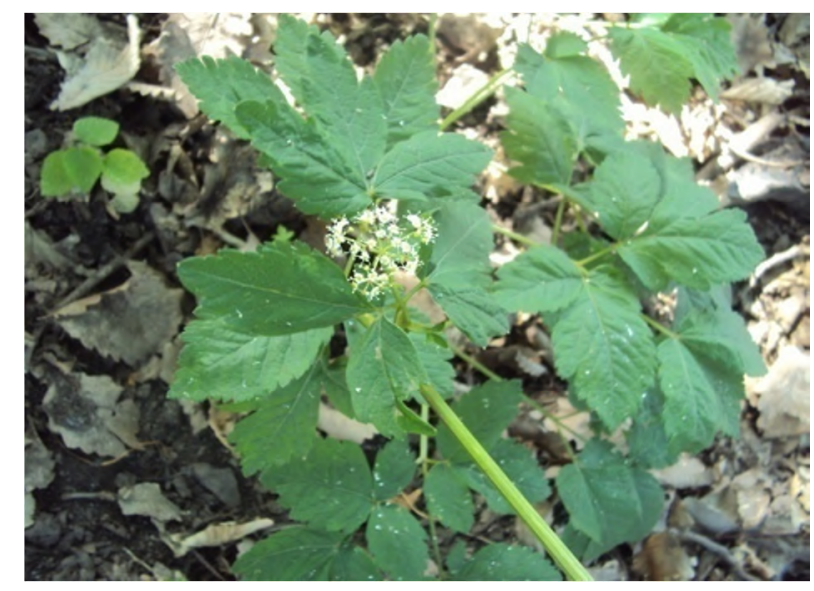

Figure 12 : Physospermum verticillatum (Waldst. \& Kit.) Vis.

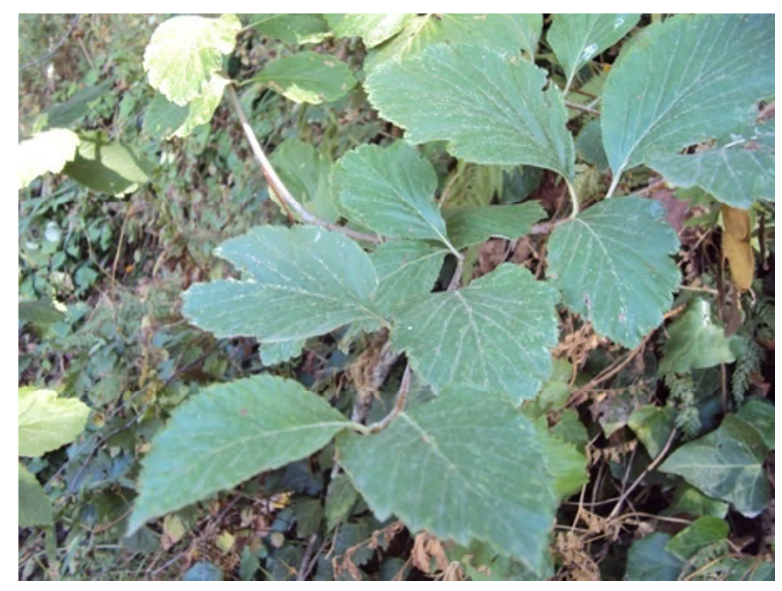

Figure 13 : Fig. 13: Sorbus aria (L.) Crantz 


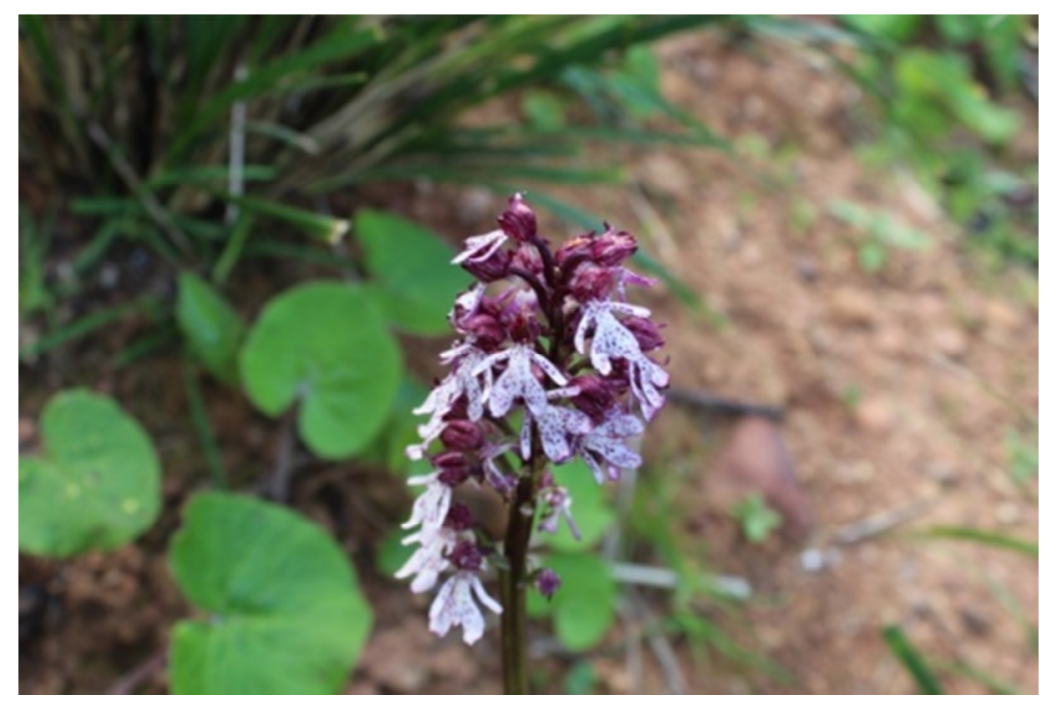

Figure 14 : Orchis purpurea Huds. subsp. purpurea

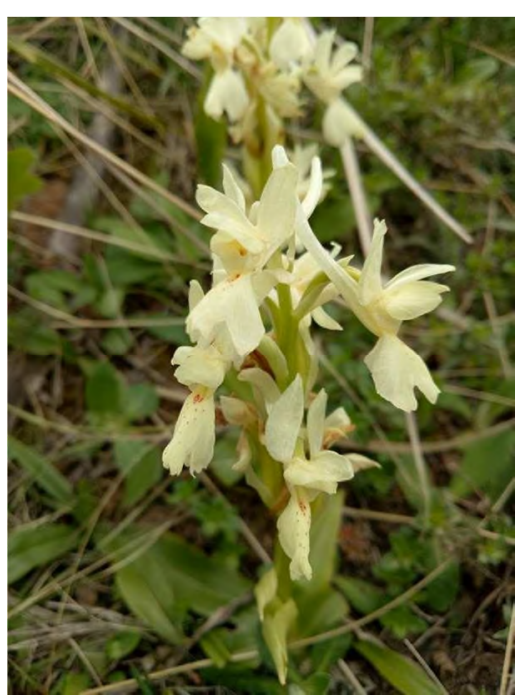

Figure 15 : Androrchis pauciflora subsp.laeta

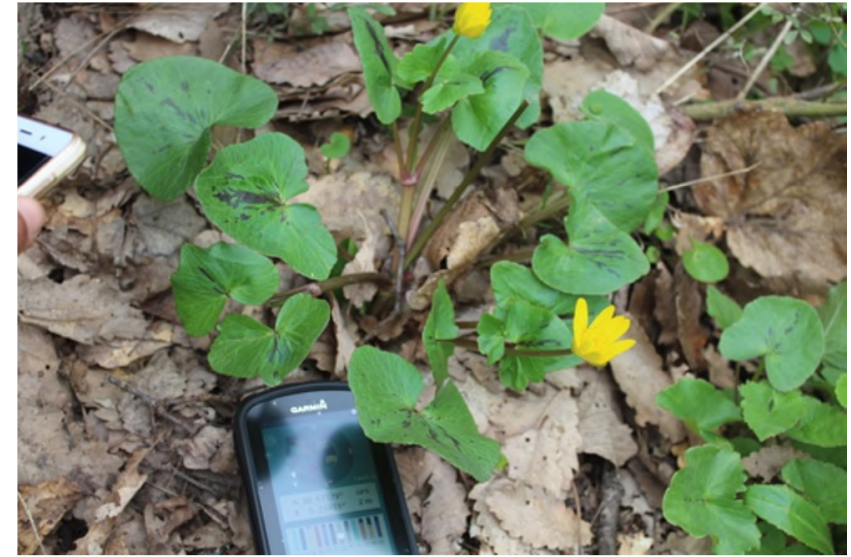

Figure 16 : Ranunculus ficariiformis (F. W.) Schultz.

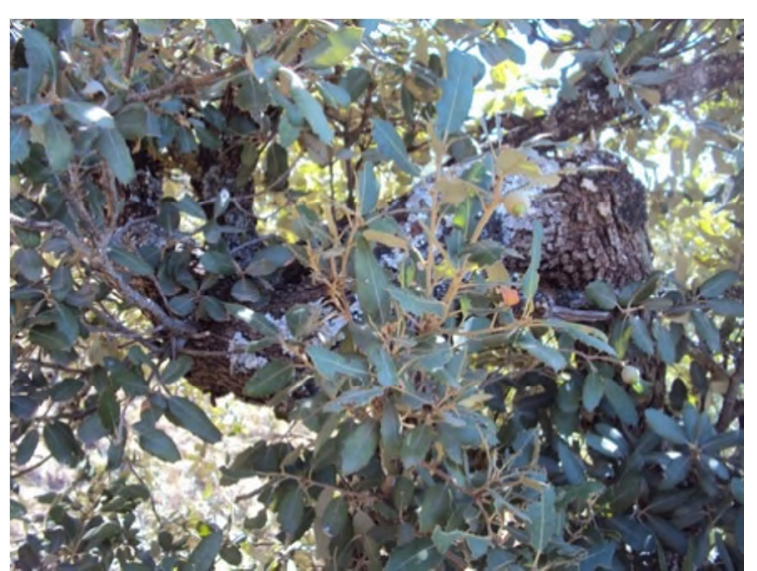

Figure 17 : Quercus ilex subsp. ballota (Desf.) Samp. 


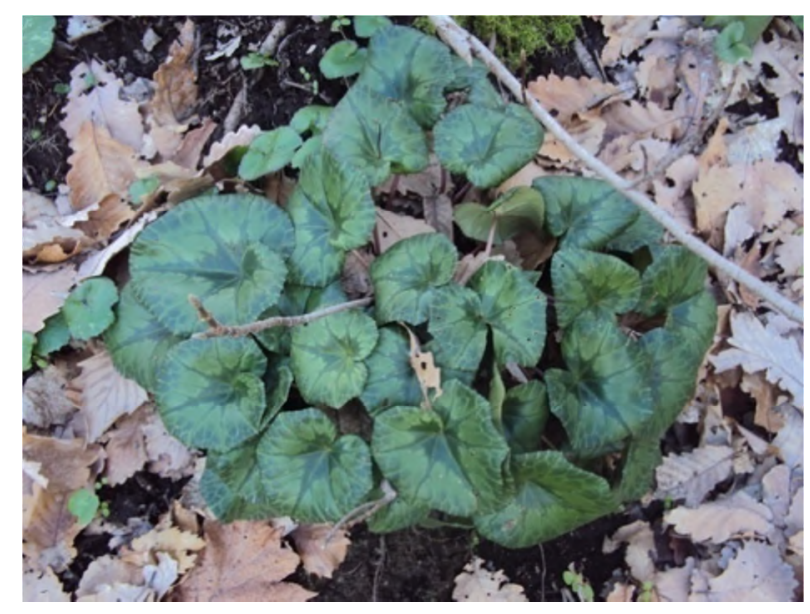

Figure 18 : Cyclamen africanum Boiss. \& Reut.

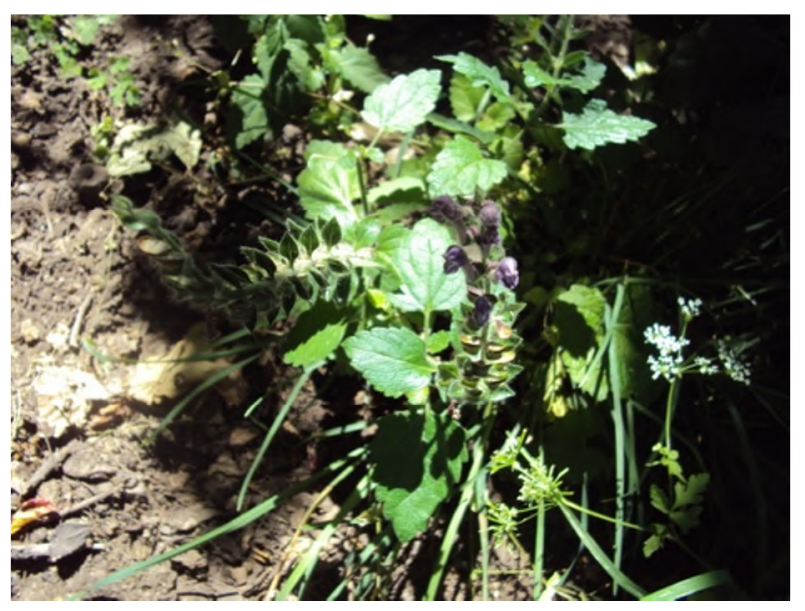

Figure 19 : Scuttelaria columnae All.

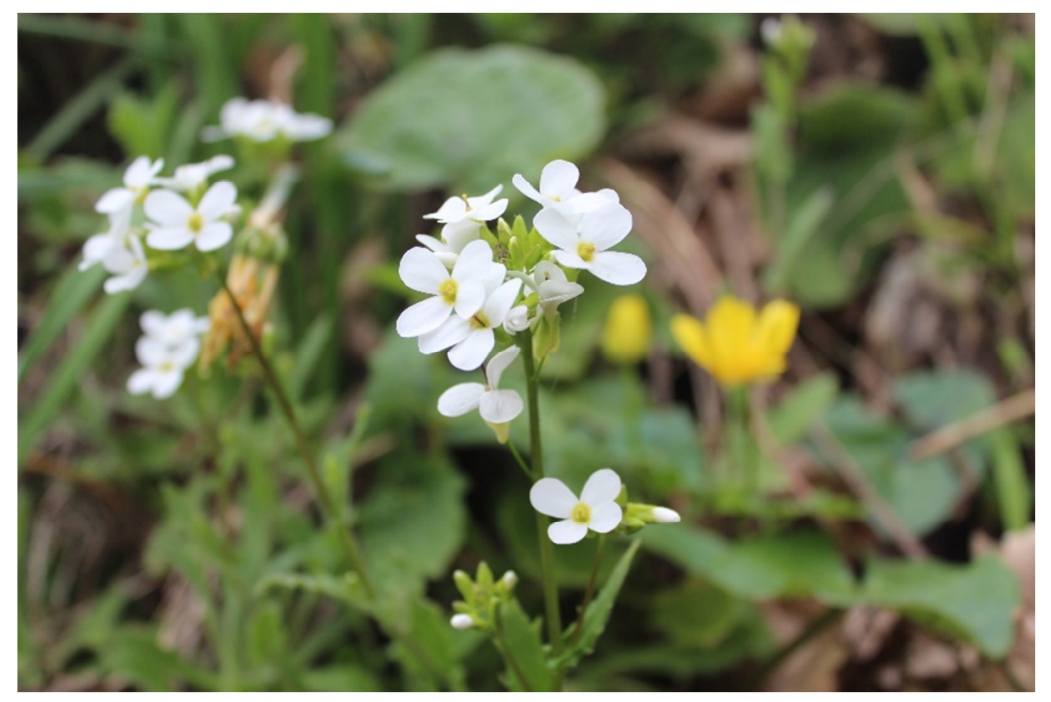

Figure 20 : Erinacea anthyllis Link

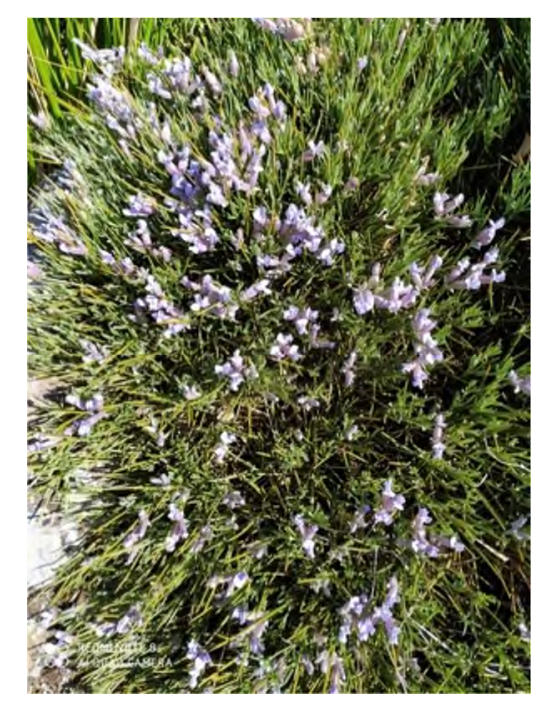

Figure 21 : Cedrus atlantica (Endl.) Carrière 
Bulletin de la Société Royale des Sciences de Liège, Vol. : 91, articles, 2021, p. 317 - 360

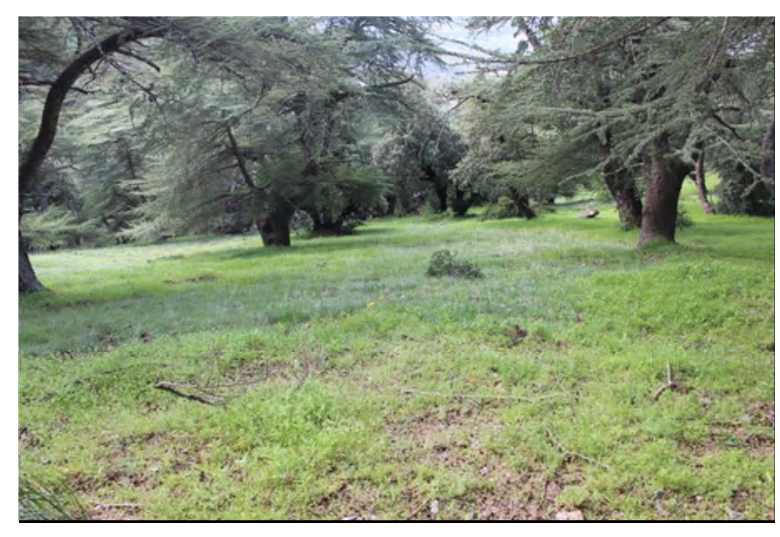

Figure 22 : Cedrus atlantica (Endl.) Carrière

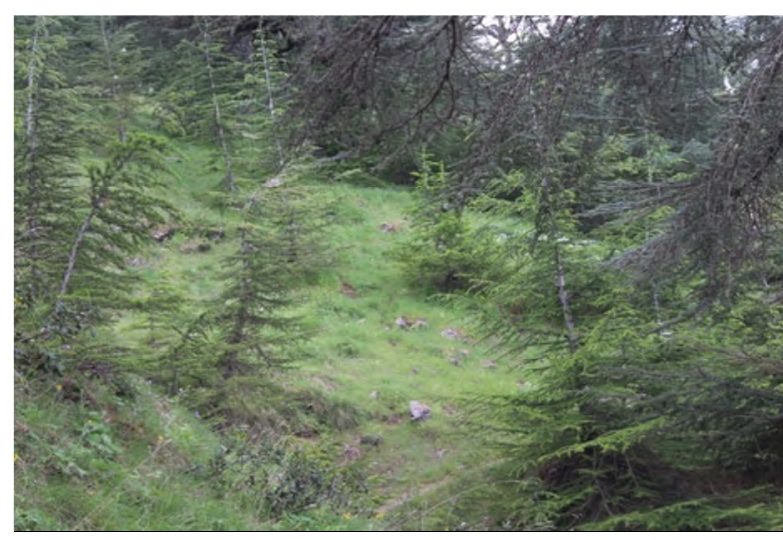

Figure 23 : Cedrus atlantica (Endl.) Carrière 